\title{
Contagious Criminal Career Models Showing Backward Bifurcations: Implications for Crime Control Policies
}

\author{
Silvia Martorano Raimundo, ${ }^{1}$ Hyun Mo Yang $\mathbb{D}^{2},{ }^{2}$ and Eduardo Massad $\mathbb{D}{ }^{1,3}$ \\ ${ }^{1}$ Faculdade de Medicina da Universidade de São Paulo, MLS and LIM01-HCFMUSP, São Paulo, SP, Brazil \\ ${ }^{2}$ Universidade de Campinas, DMA-IMECC, Campinas, SP, Brazil \\ ${ }^{3}$ School of Applied Mathematics, Fundação Getúlio Vargas, Rio de Janeiro, RJ, Brazil
}

Correspondence should be addressed to Eduardo Massad; edmassad@dim.fm.usp.br

Received 7 March 2018; Revised 30 May 2018; Accepted 3 July 2018; Published 1 August 2018

Academic Editor: Zhidong Teng

Copyright (c) 2018 Silvia Martorano Raimundo et al. This is an open access article distributed under the Creative Commons Attribution License, which permits unrestricted use, distribution, and reproduction in any medium, provided the original work is properly cited.

\begin{abstract}
We provide a theoretical framework to study how criminal behaviors can be treated as an infectious phenomenon. There are two infectious diseases like models that mimic the role of convicted criminals in contaminating individuals not yet engaged in the criminal career. Equilibrium analyses of each model are studied in detail. The models proposed in this work include the social, economic, personal, and pressure from peers aspects that can, theoretically, determine the probability with which a susceptible individual with criminal propensity engages in a criminal career. These crime-inducing parameters are treated mathematically and their inclusion in the model aims to help policy-makers design crime control strategies. We propose, to the best of our knowledge by the first time in quantitative criminology, the existence of thresholds for the stability of crime-endemic equilibrium which are the equivalent to the "basic reproduction number" widely used in the mathematical epidemiology literature. Both models presented the phenomena of backward bifurcation and breaking-point when the contact rates are chosen as bifurcation parameters. The finding of backward bifurcation in both models implies that there is an endemic equilibrium of criminality even when the threshold parameter for contagion is below unit, which, in turn, implies that control strategies are more difficult to achieve considerable impact on crime control.
\end{abstract}

\section{Introduction}

There is now substantial support in the specialized literature on economics, sociology, criminology, and social psychology to the attempts to explain how and why an individual's propensity to engage in criminal behavior is influenced by his/her social context [1]. One interesting metaphor is the one that states that criminal behavior is contagious or that individuals can be susceptible to what economists call endogenous effects [2]. According to this effect, the social milieu in which individuals live may change the individual's propensity to engage in that same criminal behavior as their peers $[3,4]$. In addition, the individual's criminal behavior can be affected by other attributes of his/her neighbors, like in the "role model" theory [5] or the peer pressure to maintain local order [6]. Moreover, the institutional or other characteristics of neighborhoods, including crime prevalence, may induce criminal behavior in susceptible individuals [7].

However, in spite of the large theoretical literature on whether or not crime is contagious, the empirical support for this hypothesis is still limited. Some authors (see $[8,9]$ ) reported an excess in the variation in crime rates across areas which cannot be explained only by the variation in standard sociodemographic determinants of criminal behavior. This suggests that social interactions are more important for less serious than more serious crimes. In a famous study, Crane (1991) (see [10]) showed that in the presence of endogenous residential sorting such reports may be biased by the causal effects of environmental and some individual or family characteristics on the selection of the neighborhood. As pointed out by Kling and Ludwig (see [1]), however, even in the absence of the biased selection problem, it would be very difficult to determine which of the theoretical perspectives 
above are responsible for any observed neighborhood effects on criminal behavior.

The influence of others' behavior on criminal offences of susceptible individuals is called "behavioral contagion" $[11,12]$. Behavioral contagion has been defined as the spread of any attitude or behavior from one individual or group of individuals to another individual or group of individuals throughout a social network of varying structures [11].

Social contagion arises among people interacting in social structures of diverse nature [13-15]. In such interactions information, behavioral innovation, belief, or meme is transmitted in a similar way to infectious diseases spread in groups of susceptible individuals [16]. Contagion occurs when susceptible individuals interact with contagious people in such a way that this interaction results in a new case [17]. In social networks, contact is defined by the communication and/or imitation of influential processes that make transmission potentially effective (see [13] pp. 1288-1289).

The social contagion theory of violence describes the spread of criminal behavior as similar to the spread of infectious diseases [18]. In such a context, the contagious nature of criminal behavior can be understood, described, and analyzed with the tools developed for studying infectious diseases spreading, in particular the use of mathematical models [19]. However, in contrast with directly transmitted diseases, the infectivity of violence does not require direct contact between susceptible and infected individuals.

In this paper, we provide a different approach to the contagious effect of criminal behavior in susceptible individuals. Rather than considering direct contagious effect by the social environment, we consider a contagious effect by criminals already convicted and who control criminal activities from inside prison, a common effect of some countries like Brazil.

The advent of organized crime in Brazilian prisons, especially in the state of São Paulo, and its role in the contagion of criminality to susceptible individuals outside the prisons constitute the object of this article. The gang leaders the Capital's First Command (PCC, Primeiro Comando da Capital) (see [20]) unleashed a series of attacks in May 2006, resulting in deaths, brought cities to a halt, and cornered authorities in charge preventing them from applying law and order and these are the starting as well as reference points taken. In addition, the gang leaders were sustained by an organization maintained by a hierarchical structure of disciplined and obedient employees capable of executing orders without questioning them. Operating from a base of support networks disseminated in distinct mobile points throughout the state, they revealed that they had an able and agile communication system among leaders, followers, and those who took orders, through protected channels barely permeated by external interference by means of cell phones, telephone exchanges, and carrier pigeons [21].

Criminal contagion from inside the prison system of Brazil to outside susceptible individuals with criminal propensity will be considered as an infectious event in a dynamical system context. The models proposed in this work include the social, economic, personal, and pressure from peers aspects that can, theoretically, determine the probability with which a susceptible individual with criminal propensity engage in a criminal career. These crime-inducing parameters are treated mathematically and their inclusion in the model aims to help policy-makers design crime control strategies.

\section{The Models}

The models presented here are based on the criminal activity within a population in an effort to understand how the criminal careers change and evolve over time. We assume a population-based approach, similar to those models of the spreading of infections that confer temporary/permanent immunity. By introducing the key epidemiological concept of a threshold from mass action law [22, 23], we illustrate the fundamental relationship between incarceration and recidivism within a population and use it to show how the criminal activity could be controlled to reduce the likelihood of an individual to engage in a criminal career. We compute and analyze this threshold considering the spread of the crime and the dynamics of incarceration and recidivism. Keeping in mind both the threshold and the perspective of criminal dynamics, it is possible to evaluate what programs of rehabilitation or prevention contribute to the reduction of the recidivism and the number of contacts among individuals susceptible to crime, offenders, and ex-offenders.

In epidemiology theory, the core groups are conceptualized as being the individuals in a population who will infect more than one person over the duration of infection. Core groups are recognized as playing a central role in sustaining the infection in a population and interventions targeting these groups are central to an effective prevention response. Building on this, we extend the concept of core groups to the inmate population.

Especially in inmate population, most criminality is found among core groups and the criminality will only become more generalized if the contact spreads throughout other networks. Hence, it is very likely that incarcerated individuals will contact those susceptible individuals who have never been incarcerated but have an intrinsic criminal propensity. The basic problem is to find out when this contact occurs, who regulates contact, what types of contact are feasible and desirable, and what are the effects of contact (or lack thereof) on susceptible individuals.

In this way, the total population size, denoted by $N(t)$, is then characterized by three classes: susceptible $(S)$, incarcerated $(C)$, and desisting $(D)$ individuals. We specify firsttime $(\mathrm{i}=1)$ and multiple-time $(\mathrm{i}=2,3, \ldots, \mathrm{n})$ incarceration by adding subscripts to model variables and parameters.

\subsection{The Partially Contagious Criminality Model (PCCM)} Formulation. Let the susceptible in core group population be divided into two categories: $S_{0}$, those individuals who have a criminal propensity but have never been incarcerated, not criminally active but susceptible to crime, and $S_{i}$, those individuals susceptible to criminal activities who were once incarcerated $\left(S_{1}\right)$ and those susceptible who were multipletime incarcerated $\left(S_{i}, i=2,3, \ldots, n\right)$ and became criminally active again. Similarly, let the incarcerated population be divided into two categories: $C_{1}$, those who are first-time 
incarcerated, and $C_{i}(\mathrm{i}=2,3, \ldots, \mathrm{n})$, those who were multipletime incarcerated at a given time. Finally, $D_{0}$ represents those individuals who have a criminal propensity but desisted from criminal life either by their own or as a result of early interventions when discharged out of prison (desisting offenders/criminal desisters), and $D_{i}(\mathrm{i}=1,2,3, \ldots, \mathrm{n})$ represents those individuals who were either first-time or multiple-time incarcerated but desisted from criminal behavior (desisting offenders/criminal desisters) due to variety of reasons.

It is assumed that individuals susceptible to the crime $S_{0}$ move to either incarcerated $\left(C_{1}\right)$ or desisting $\left(D_{0}\right)$ class. The rate of initial participation in crime $\left(\delta_{0}\right)$ at which individuals move from state $S_{0}$ (not criminally active) to $C_{1}$ (criminally active and incarcerated) is proportional to intrinsic criminal propensity of individuals. It is also assumed that special intervention programmes $\left(\gamma_{1}\right)$ may change their basic propensity traits and affect the decisions to engage in crime [24]. As a consequence, we should expect that certain interventions discourage participation in crime, resulting in desistance from criminal activity and reintegration back into society. Primary prevention is an attempt to reduce the risk of behaviors that potentially lead to incarceration. This point is one of the great interests here.

The average length of the primary incarceration term is given by $1 / \tau_{1}$; that is, the rate at which inmates move from state $C_{1}$ to $S_{1}$ (formerly incarcerated but not criminally active, i.e., ex-offenders) is $\tau_{1}$. In the same way, the average length of the multiple incarceration term is given by $1 / \tau_{2}$, with $1 / \tau_{2}>$ $1 / \tau_{1}$.

It is worth mentioning that the representation of the cycles of criminal dynamics, with a focus on modeling the recidivism process (criminal careers), could be extended including $S_{i}, C_{i}$, and $D_{i}(\mathrm{i}=2,3, \ldots, \mathrm{n})$. However, we do not intend to develop complex models that account for these cycles and we will explore two cycles only $(i=1,2)$.

In addition, it is assumed that the recidivism prevention takes place during incarceration and after release back into society. Its purpose is to reduce the risk of an individual reoffending and eventually returning to the prison system. However, some individuals may resume criminal activity very soon after being released from prisons depending on having contact with those individuals still incarcerated and criminally active. It should be mentioned that Walsh and Graig (see [25]) indicated that recidivism is also called falling back into a previous criminal behavior.

We also define $\beta_{i}(\mathrm{i}=1,2)$ as the rate of imprisonment, which captures the return to criminal activity of the individuals released from prison, such that $\beta_{1}$ and $\beta_{2}$ describe the rates with which individuals engage into criminal activity, depending on having had any contagious contact with those incarcerated criminals. They are analogous to the effective contact rate in infectious diseases models.

Finally, $\mu$ is the natural mortality rate and $a_{i}(\mathrm{i}=1,2)$ is the incarceration-related additional deaths rate (inmate mortality rate can be caused by illness, such as AIDS-related, suicide, accidental self-injury, execution, or any other unspecified cause). Moreover, since the model monitors human populations, all parameters are assumed as nonnegative. We also assumed homogeneous population without any differences in

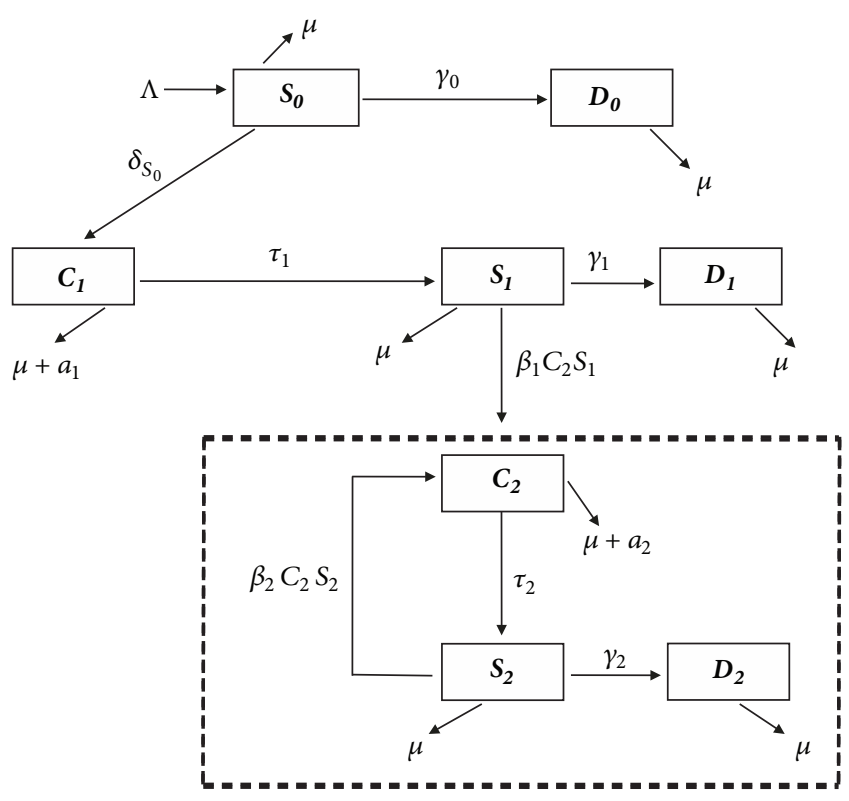

FIgURE 1: The flow diagram for the low-high criminality model (1).

age or in crime type occurrences. In addition, we used the number of incarcerations as a proxy for reoffending.

The flow diagram of the PCCM for two stages is depicted in Figure 1. The variables and parameters are described in Tables 1 and 2, respectively.

Combining the above derivations and assumptions, it follows that the model for transmission dynamics of criminality for two stages is given by the following nonlinear system of differential equations:

$$
\begin{aligned}
\frac{d S_{0}}{d t} & =\Lambda-\left(\delta_{0}+\gamma_{0}+\mu\right) S_{0} \\
\frac{d D_{0}}{d t} & =\gamma_{0} S_{0}-\mu D_{0} \\
\frac{d C_{1}}{d t} & =\delta_{0} S_{0}-\left(\tau_{1}+\mu+a_{1}\right) C_{1} \\
\frac{d S_{1}}{d t} & =\tau_{1} C_{1}-\left(\gamma_{1}+\mu\right) S_{1}-\beta_{1} C_{2} S_{1} \\
\frac{d D_{1}}{d t} & =\gamma_{1} S_{1}-\mu D_{1} \\
\frac{d C_{2}}{d t} & =\left(\beta_{1} S_{1}+\beta_{2} S_{2}\right) C_{2}-\left(\tau_{2}+\mu+a_{2}\right) C_{2} \\
\frac{d S_{2}}{d t} & =\tau_{2} C_{2}-\beta_{2} C_{2} S_{2}-\left(\gamma_{2}+\mu\right) S_{2} \\
\frac{d D_{2}}{d t} & =\gamma_{2} S_{2}-\mu D_{2}
\end{aligned}
$$

with generic initial conditions $S_{0}(0) \geq 0, D_{0}(0) \geq 0, C_{1}(0) \geq$ $0, S_{1}(0) \geq 0, D_{1}(0) \geq 0, C_{2}(0) \geq 0, S_{2}(0) \geq 0$, and $D_{2}(0) \geq 0$. 
TABLE 1: Models variables and their biological meaning.

\begin{tabular}{ll}
\hline Variables & Description \\
\hline$S_{0}$ & Individuals not criminally active but susceptible to crime (core group) \\
$C_{1}$ & First-time incarcerated individuals \\
$S_{1}$ & First-time ex-offenders individuals who are again susceptible to crime \\
$D_{i}$ & Individuals who desist from criminal behavior (i = 0,1,2) (desisting offenders/criminal desisters) \\
$C_{2}$ & Individuals who were two or multiple times incarcerated (recidivists, reoffenders) \\
$S_{2}$ & Second-time ex-offenders individuals and susceptible to crime (at least two incarcerations) \\
\hline
\end{tabular}

TABLE 2: Models parameters and their biological meaning.

\begin{tabular}{ll}
\hline Parameters & Description \\
\hline$\Lambda$ & Rate of recruitment of individuals into the core group \\
$\delta_{0}$ & Basic flow to the criminality $\left(\right.$ time $\left.^{-1}\right)($ criminal propensity) \\
$\beta_{1}$ & Rate of cooptation of first-time ex-offenders $\left(\right.$ contact rate between $C_{2}$ and $\left.S_{1}\right)$ \\
$\beta_{2}$ & Rate of cooptation of second-time ex-offenders $\left(\right.$ contact rate between $C_{2}$ and $\left.S_{2}\right)$ \\
$\gamma_{0}$ & Rate of early desistance from crime \\
$\gamma_{1}$ & Rate of desistance from crime when in the first cycle \\
$\gamma_{2}$ & Rate of desistance from crime when in at least the second cycle \\
$\mu$ & Natural inmate mortality rate \\
$a_{1}$ & Additional inmate mortality rate when in $C_{1}$ \\
$a_{2}$ & Additional inmate mortality rate when in $C_{2}$ \\
$\tau_{1}$ & Release rate from incarceration of first-time offenders \\
$\tau_{2}$ & Release rate from incarceration of at least second-time offenders \\
\hline
\end{tabular}

By summing up the above equations, the total population size $N(t)$ is variable with

$$
\frac{d N}{d t}=\Lambda-\mu N-a_{1} C_{1}-a_{2} C_{2}
$$

Thus, in the absence of additional inmate mortalities, that is, $a_{1}=a_{2}=0$, the population size evolves as an immigration model with natural mortality, that is, according to $d N / d t=$ $\Lambda-\mu N$. This equation has a single equilibrium $N=N(0)=$ $\Lambda / \mu$ for any initial value of $N(0)$. Thus, in the long run, the population size settles to this constant value. It follows from (2) that $\lim _{t \rightarrow \infty} N(t) \leq \Lambda / \mu=N(0)$.

The differential equation for $N$ implies that solutions of (1), starting in the positive orthant $\mathbb{R}_{+}^{8}$, either approach, enter, or remain in the subset $\mathbb{R}_{+}^{8}$ defined by

$$
\begin{aligned}
\Omega= & \left\{\left(S_{0}, D_{0}, C_{1}, S_{1}, D_{1}, C_{2}, S_{2}, D_{2}\right) \in \mathbb{R}_{+}^{8}: S_{0}+D_{0}\right. \\
& \left.+C_{1}+S_{1}+D_{1}+C_{2}+S_{2}+D_{2} \leq N(0)\right\}
\end{aligned}
$$

Thus it suffices to consider solutions in the region $\Omega$. Solutions of the initial value problem starting in $\Omega$ and defined by (1) exist and are unique on a maximal interval [26]. Since solutions remain bounded in the positively invariant region $\Omega$, the initial value problem is then both mathematically and epidemiologically well posed [27]. Hence, it is sufficient to consider the dynamics of the flow generated by model (1) in $\Omega$.
2.2. Analysis of the PCCM Model. In this section, system (1) is qualitatively analyzed to investigate the existence of its equilibria [28] and the control strategies of its dynamical behavior.

From system (1), with the right-hand size equal to zero, it can be seen from the first five equations that the coordinates of the equilibrium point are given, respectively, by

$$
\begin{aligned}
S_{0} & =\frac{\Lambda}{\left(\delta_{0}+\gamma_{0}+\mu\right)}, \\
D_{0} & =\frac{\gamma_{0}}{\mu} S_{0} \\
C_{1} & =\frac{\delta_{0}}{\left(\tau_{1}+\mu+a_{1}\right)} S_{0}, \\
S_{1} & =\frac{\tau_{1} \delta_{0}}{\left(\tau_{1}+\mu+a_{1}\right)\left(\mu+\gamma_{1}+\beta_{1} C_{2}\right)} S_{0}, \\
D_{1} & =\frac{\gamma_{1}}{\mu} S_{1} .
\end{aligned}
$$

Moreover, from seventh and eighth equations of system (1), we obtain

$$
\begin{aligned}
S_{2} & =\frac{\tau_{2} C_{2}}{\mu+\gamma_{2}+\beta_{2} C_{2}}, \\
D_{2} & =\frac{\gamma_{2}}{\mu} S_{2} .
\end{aligned}
$$


From the sixth equation of system (1), one gets

(i) $C_{2}=0$,

(ii) $C_{2} \neq 0$, which implies $\beta_{1} S_{1}+\beta_{2} S_{2}-\left(\tau_{2}+\mu+a_{2}\right)=0$.

If $C_{2}=0$, model (1) has a low-criminality equilibrium $P_{l}=\left(S_{0}, D_{0}, C_{1}, S_{1}, D_{1}, 0,0,0\right)$ which indicates the existence of offenders who are incarcerated only once in life $\left(C_{1}\right)$, given by

$$
\begin{aligned}
S_{0} & =\frac{\Lambda}{\left(\delta_{0}+\gamma_{0}+\mu\right)}, \\
D_{0} & =\frac{\gamma_{0}}{\mu} S_{0}, \\
C_{1} & =\frac{\delta_{0}}{\left(\tau_{1}+\mu+a_{1}\right)} S_{0}, \\
S_{1} & =\frac{\tau_{1} \delta_{0}}{\left(\tau_{1}+\mu+a_{1}\right)\left(\mu+\gamma_{1}\right)} S_{0}, \\
D_{1} & =\frac{\gamma_{1}}{\mu} S_{1} .
\end{aligned}
$$

To determine the stability of this equilibrium, the Jacobian of system (1) is computed and evaluated at $P_{l}$. Hence, the low-criminality equilibrium $P_{l}=\left(S_{0}, D_{0}, C_{1}, S_{1}, D_{1}, 0,0,0\right)$ is locally asymptotically stable if $R_{1}^{*}<1$, where $R_{1}^{*}$, defined as Criminality Reproduction Number (CRN), is given by

$$
R_{1}^{*}=\frac{\beta_{1}}{\beta_{1}^{*}}
$$

with

$$
\beta_{1}^{*}=\frac{\left(\tau_{1}+\mu+a_{1}\right)\left(\mu+\gamma_{1}\right)\left(\tau_{2}+\mu+a_{2}\right)}{\tau_{1} \delta_{0} S_{0}} .
$$

Note that, in analogy to the spread of infectious diseases models [29], the CRN, $R_{1}^{*}$, represents the "average expected number of new offenders originated by a single persisting offender $C_{2}^{*}$, whilst in a criminal career." In other words, one person $C_{2}^{*}$ who was incarcerated two times gets into contact with $S_{1}$ susceptible individuals, just released from first imprisonment, successfully and induces $R_{1}^{*}$ persons to commit crime. In other words, $R_{1}^{*}$ is the average number of individuals who commit crime influenced by one inmate $C_{2}^{*}$.

For $C_{2} \neq 0$, that is, for $\beta_{1} S_{1}+\beta_{2} S_{2}-\left(\tau_{2}+\mu+a_{2}\right)=0$, replacing both expressions for $S_{1}$ given by (4) and $S_{2}$ given by (5), an expression for $C_{2}=C_{2}^{*}>0$ is obtained as

$$
b_{2}\left(C_{2}^{*}\right)^{2}+b_{1} C_{2}^{*}+b_{0}=0
$$

where

$$
\begin{aligned}
b_{2} & =\beta_{1} \beta_{2}\left(\mu+a_{2}\right) . \\
b_{1} & =\left(\mu+\gamma_{1}\right)\left(\tau_{2}+\mu+a_{2}\right) \\
& \cdot \beta_{2}\left\{\left[\frac{\beta_{1}\left(\mu+\gamma_{2}\right)}{\beta_{2}\left(\mu+\gamma_{1}\right)}+\frac{\left(\mu+a_{2}\right)}{\left(\tau_{2}+\mu+a_{2}\right)}\right]-R_{1}^{*}\right\}, \\
b_{0} & =\left(\mu+\gamma_{1}\right)\left(\mu+\gamma_{2}\right)\left(\tau_{2}+\mu+a_{2}\right)\left(1-R_{1}^{*}\right),
\end{aligned}
$$

Let us now determine the conditions under which the quadratic equation (9) has positive real roots; that is, we search for the existence of multiple equilibria of system (1). However, the expression for the discriminant of the quadratic equation (9) is very complex, so we will analyze the signs of its coefficients to ensure the existence of real solutions. Thus, the conditions under which this equation has either one or two positive real roots can be determined, and these results translated into nontrivial equilibrium of system (1) which is biologically feasible (i.e., positive).

Hence, substituting the positive real solutions of the quadratic equation (9) (i.e., positive values of $C_{2}^{*}$ ) into the expressions in (5), model (1) has a high-criminality equilibrium, $P_{h}=\left(S_{0}, D_{0}, C_{1}, S_{1}^{*}, D_{1}^{*}, C_{2}^{*}, S_{2}^{*}, D_{2}^{*}\right)$, where there is coexistence of both offenders $C_{1}$ and $C_{2}^{*}$, given by

$$
\begin{aligned}
& S_{1}^{*}=\frac{\tau_{1} \delta_{0}}{\left(\tau_{1}+\mu+a_{1}\right)+\left(\mu+\gamma_{1}\right)+\beta_{1} C_{2}^{*}} S_{0} \\
& D_{1}^{*}=\frac{\gamma_{1}}{\mu} S_{1}^{*} \\
& S_{2}^{*}=\frac{\tau_{2} C_{2}^{*}}{\left(\mu+\gamma_{2}\right)+\beta_{2} C_{2}^{*}} \\
& D_{2}^{*}=\frac{\gamma_{2}}{\mu} S_{2}^{*},
\end{aligned}
$$

with $S_{0}, D_{0}$, and $C_{1}$ given by (6). Thus, the following result is then established.

Theorem 1. Model (1) has

(i) a unique positive equilibrium $P_{h}$ if $b_{0}<0 \Longleftrightarrow R_{1}^{*}>$ 1 ;

(ii) a unique positive equilibrium $P_{h}$ if $b_{0}=0$ and $b_{1}<0$;

(iii) two positive equilibria, $P_{h}$, if $b_{0}>0$ and $b_{1}<0$ and $b_{1}^{2}-4 b_{2} b_{0}>0$

(iv) no positive equilibrium, otherwise.

Since all model parameters are assumed as nonnegative, it follows from (10) that the coefficient $b_{2}$ is always positive, $b_{0}<0$ for $R_{1}^{*}>1$ and $b_{0}>0$ for $R_{1}^{*}<1$. Thus, it is clear from Theorem 1 that model (1) has a unique positive equilibrium, $P_{h}$, when $b_{0}<0$, that is, when $R_{1}^{*}>1$ (case (i)).

Now, for $b_{0}>0$ and $R_{1}^{*}<1$, the quadratic equation (9) has two positive solutions if $b_{1}<0$ and $b_{1}^{2}-4 b_{2} b_{0}>0$ (case (iii)). Hence, assuming that (9) has two positive real solutions, let $C_{2}^{-}$and $C_{2}^{+}$be the smaller and higher value of $C_{2}^{*}$, respectively. Translating it into equilibrium of system (1), the question is to address what means the positive high-criminality equilibria $P_{h}$ for $R_{1}^{*}<1$. It is important to note that, in this case, system (1) can have two equilibria, which are biologically feasible, even though $R_{1}^{*}<1$. This idea is explored more deeply below.

It is instructive at this point to explore some qualitative features for $R_{1}^{*}=1$ and $R_{1}^{*}>1$. Firstly, for $R_{1}^{*}=1$, it follows that $b_{0}=0$; the quadratic equation (9) has either a unique positive root (if $b_{1}<0$ ) or no positive root (if $b_{1}>0$ ). In other words, for $R_{1}^{*}=1$ and $b_{1}<0$, model (1) has a unique positive high-criminality equilibrium given by $P_{h}$ (case (ii)). 
Moreover, note that when $\beta_{2}=0$, we have $b_{2}=0$ and $b_{1}=\left(\mu+\gamma_{2}\right)\left(\tau_{2}+\mu+a_{2}\right) \beta_{1}$. Thus, if $R_{1}^{*}>1$, then

$$
C_{2}^{*}\left(\beta_{2}=0\right)=\frac{\left(\mu+\gamma_{1}\right)}{\beta_{1}}\left(R_{1}^{*}-1\right),
$$

and model (1) has a unique positive high-criminality equilibrium, $P_{h}$, for $\beta_{2}=0$.

For $\beta_{2} \longrightarrow \infty$, we have $b_{1}=\left(\mu+\gamma_{1}\right)\left(\tau_{2}+\mu+a_{2}\right) \beta_{2}[(\mu+$ $\left.\left.a_{2}\right) /\left(\tau_{2}+\mu+a_{2}\right)-R_{1}^{*}\right]$ and $b_{2}=\beta_{1} \beta_{2}\left(\mu+a_{2}\right)$ with $b_{0} \ll\left|b_{1}\right|$ and $b_{0} \ll b_{2}$ such that

$$
\begin{aligned}
C_{2}^{*} & \left(\beta_{2} \longrightarrow \infty\right) \\
& =\frac{\left(\mu+\gamma_{1}\right)\left(\tau_{2}+\mu+a_{2}\right)}{\beta_{1}\left(\mu+a_{2}\right)}\left[R_{1}^{*}-\frac{\left(\mu+a_{2}\right)}{\left(\tau_{2}+\mu+a_{2}\right)}\right],
\end{aligned}
$$

and $C_{2}^{*}>0$ if only if $R_{1}^{*}>\left(\mu+a_{2}\right) /\left(\tau_{2}+\mu+a_{2}\right)$. Thus, model (1) has a unique high-criminality equilibrium $P_{h}$ if $\beta_{2} \rightarrow \infty$.

Unfortunately, this high-criminality equilibrium cannot be studied from its closed form, so we carried out its local stability using numerical methods. The results are provided next.

Finally, if (i), (ii), and (iii) do not occur, then there are no endemic equilibria for system (1).

In what follows, model (1) admits two realistic scenarios: the best-case scenario, where offenders were incarcerated once and it is still possible to fight crime and recidivism is given by the low-criminality equilibrium $P_{l}=$ $\left(S_{0}, D_{0}, C_{1}, S_{1}, D_{1}, 0,0,0\right)$, and the worst-case scenario, where the offenders were incarcerated at least once (recidivism), which can potentially lead to increased criminal activities, given by the high-criminality equilibrium $P_{h}=$ $\left(S_{0}, D_{0}, C_{1}, S_{1}^{*}, D_{1}^{*}, C_{2}^{*}, S_{2}^{*}, D_{2}^{*}\right)$.

Having found the scenarios in which there exist the equilibria for system (1), it is instructive to analyze whether or not these equilibria are stable under any of these scenarios. Moreover, together with the CRN, $R_{1}^{*}$ (see (7)), and the parameter $\beta_{2}$, we see that each scenario can be used as a check for the existence and the stability of the equilibria. Another crucial question is which of the two incidence rates $\beta_{1}$ and $\beta_{2}$ is more likely to affect the criminal dynamics in general and criminal contacts in particular. These points are of great interest here and we then explore how our model behaves under control strategies when $R_{1}^{*}<1$. We fix $\beta_{2}$ and explore the system behavior by varying $\beta_{1}$. We also explore what happens when we fix $\beta_{1}$ while $\beta_{2}$ is varied. These control measures are designed to fight recidivism. In both cases, the recidivism will depend upon the initial sizes of the subpopulation $C_{2}$ and indicate the possibility of backward bifurcation.

Firstly, it is worth remembering that the quantity $\beta_{1}$ measures the average number of new contacts generated by a typical incarcerated individual $C_{2}$ with those susceptible individuals $S_{1}$ who are ex-offenders and can become susceptible to the crime again. $\beta_{2}$, in turn, measures the average number of new contacts generated by $C_{2}$ with those individuals $S_{2}$ who were reoffenders (recidivist behavior) and are susceptible to the crime once again.
In this way, there are two groups of offenders: those who are under much lower risk, most of whom will go to prison once and not come back (ex-offenders), and those who repeatedly do crimes and come back multiple times to the prison (reoffenders). The strong implication of the findings is that individuals who are incarcerated are extremely likely to reoffend once they are free.

Note that when system (1) has a small influx of reoffenders $\left(C_{2}\right)$, it does not generate high criminality rates and, for $R_{1}^{*}<1$, it is still possible to minimize the spreading of crime (best-case scenario). We will see that, in this case, the corresponding low-criminality equilibrium $P_{l}$ could be locally asymptotically stable. On the other hand, the criminality will persist and increase if $R_{1}^{*}>1$ (worst-case scenario). In this case, the corresponding high-criminality equilibrium $P_{h}$ could be locally asymptotically stable. This phenomenon, where the possibility of fighting the spread of the crime is lost and the criminality is potentially active, that is, where $P_{l}$ loses its stability and a unique $P_{h}$ appears as $R_{1}^{*}$ increases through one, is known as forward bifurcation in epidemiology.

For models that exhibit this type of bifurcation, the requirement $R_{1}^{*}<1$ is necessary and sufficient for the highcriminality elimination. In contrast, other models undergo another type of bifurcation, known as "backward bifurcation" in epidemiology, where two equilibria $P_{h}^{+}$and $P_{h}^{-}$coexist with the low-criminality equilibrium, $P_{l}$; that is, there are three steady states when $R_{1}^{*}$ is immediately less than one. Thus, the requirement $R_{1}^{*}<1$ is necessary but is not sufficient to fight the spread of crime.

2.2.1. Analytic Strategy: $\beta_{2}$ Fixed with $\beta_{1}$ Increasing. The physical implication of backward bifurcation is that the $C_{2}$ population can engage in a high criminality level even when $R_{1}^{*}$ crosses unity downwards. In other words, in the presence of recidivism, decreasing $R_{1}^{*}$ below one is not a sufficient condition to make the criminality level decrease. This has very important consequence for crime control, as will be discussed later in this paper.

To check the possibility of backward bifurcation in model (1), it is necessary to know other subthreshold, and we will refer to this limit point, as expressed on the $R_{1}^{*}$ scale, as $R_{1}^{t h r}$. Hence, for $R_{1}^{*}<R_{1}^{t h r}$, system (1) will present only the locally asymptotically stable low-criminality equilibrium $P_{l}$. For $R_{t h r}^{*}<R_{1}^{*}<1$, system (1) will present the locally asymptotically stable low-criminality equilibrium $P_{l}$ plus two positive high-criminality equilibria, $P_{h}^{+}$and $P_{h}^{-}$, which will correspond to the solutions of (9): $C_{2}^{+}$, the higher solution, which corresponds to the stable equilibrium, and $C_{2}^{-}$, the smaller solution, which corresponds to the unstable equilibrium.

Although the critical value of the bifurcation could not be found analytically due to the high dimension of system (1), this task can be performed numerically. In this way, our simulations show that there exists a critical value $R_{t h r}^{*}<R_{1}^{*}=$ 1 , where model (1) undergoes backward bifurcation.

A schematic diagram of the backward and the forward bifurcations for system (1) is given in Figure 2, where $\beta_{1}$ is chosen as a bifurcation parameter; that is, we fixed $\beta_{2}$, whereas $\beta_{1}$ increases. 


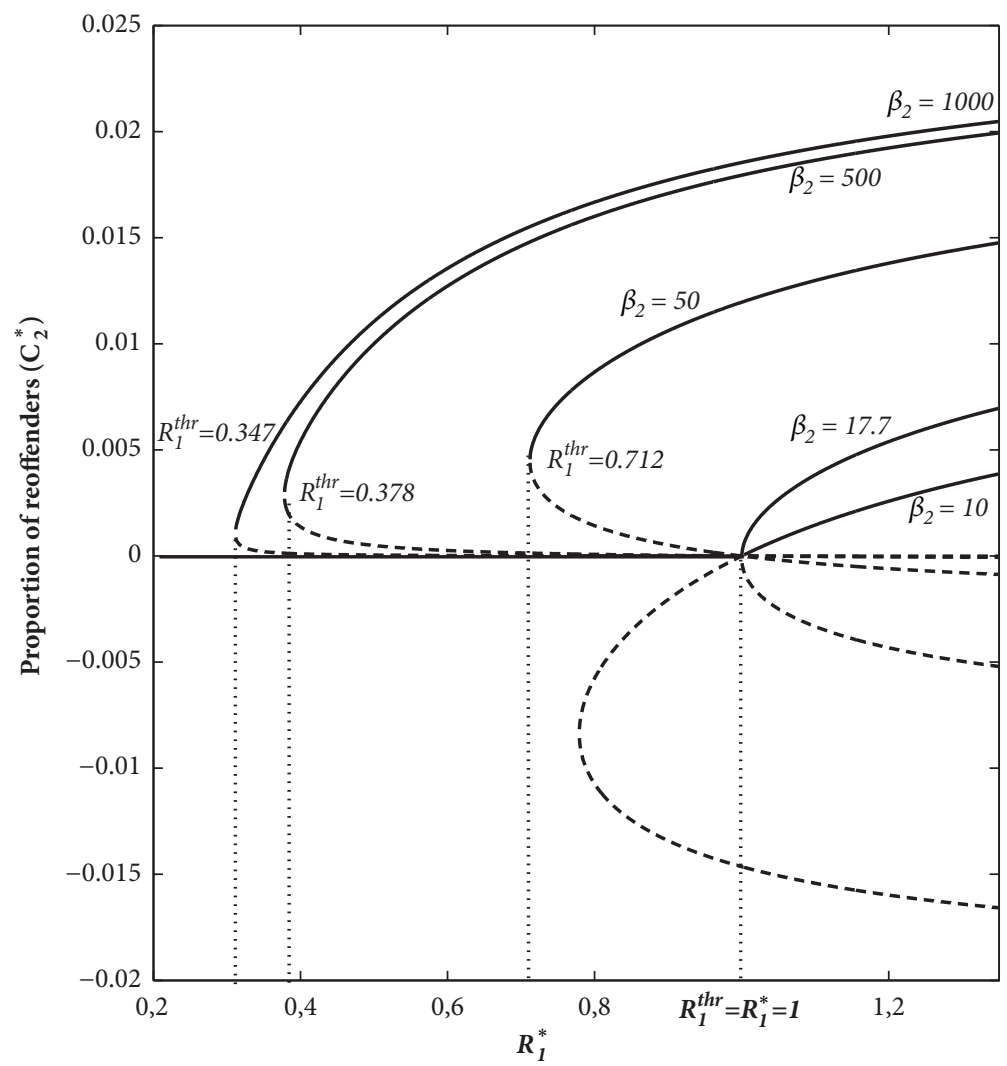

FIGURE 2: Backward and forward bifurcations diagram for proportion of reoffenders $\left(C_{2}^{*}\right)$ for model (1), where $\beta_{1}$ is chosen as a bifurcation parameter ( $\beta_{2}$ fixed and $\beta_{1}$ increasing). The higher solution corresponds to the stable equilibrium (solid curve); the smaller solution corresponds to the unstable equilibrium (dashed curve). Backward bifurcation for (a) $\beta_{2}=1000, \beta_{1}=5.371$, and $R_{1}^{\text {thr }}=0.347$; (b) $\beta_{2}=500$, $\beta_{1}=5.845$, and $R_{1}^{t h r}=0.378$; and (c) $\beta_{2}=50, \beta_{1}=11$, and $R_{1}^{t h r}=0.712$. Forward bifurcation for (a) $\beta_{2}=17.7, \beta_{1}=15.45$, and $R_{1}^{t h r}=R_{1}^{*}=1$ and (b) $\beta_{2}=10.0, \beta_{1}=15.45$, and $R_{1}^{\text {thr }}<R_{1}^{*}=1$. Parameters' values used are as given in Table 3 .

Figure 2 shows the profile of the proportion of both reoffenders $C_{2}^{+}$(solid curve) and $C_{2}^{-}$(dashed curve) as a function of $R_{1}^{*}$ with decreasing values of $\beta_{2}=1000 ; 500 ; 50 ; 17.7 ; 10\left(\right.$ year $\left.^{-1}\right)$ as $R_{1}^{*}$ increases (i.e., as $\beta_{1}$ increases). The solid curve stands for the stable high-criminality equilibrium, $C_{2}^{+}$, and the dashed curve stands for the unstable high-criminality equilibrium, $C_{2}^{-}$. For $\beta_{2}=1000$ and $\beta_{1}=5.371$, one has $R_{1}^{\text {thr }}=0.347$. Thus, for $0.347<R_{1}^{*}<1$, model (1) has two positive high-criminality equilibria, while for $R_{1}^{*}>1$, model (1) has one positive high-criminality equilibrium. Similarly, for $\beta_{2}=500$ and $\beta_{1}=5.845$, one gets $R_{1}^{t h r}=0.378$. Thus, for $0.378<R_{1}^{*}<1$, model (1) has two positive high-criminality equilibria, while for $R_{1}^{*}>1$, model (1) has one positive high-criminality equilibrium. For $\beta_{2}=50$ and $\beta_{1}=11$, one gets $R_{1}^{t h r}=0.712$. Thus, for $0.712<R_{1}^{*}<1$, model (1) has two positive high-criminality equilibria, while for $R_{1}^{*}>1$, model (1) has one positive high-criminality equilibrium. Finally, for $\beta_{2}=17.7$ and $\beta_{1}=15.45$ and for $\beta_{2}=10.0$ and $\beta_{1}=15.45$, we have $R_{1}^{\text {thr }} \leq R_{1}^{*}=1$, and the model has one positive high-criminality equilibrium for $R_{1}^{*}>1$ and no positive equilibrium for $R_{1}^{*}<1$. Consequently, model (1) exhibits the forward bifurcation at $R_{1}^{*}=1$. As it should be expected, it
TABLE 3: Baseline values for model (1).

\begin{tabular}{ll}
\hline Variable & Description \\
\hline$\Lambda$ & $0.015\left(\right.$ years $\left.^{-1}\right)$ \\
$\delta_{0}$ & $0.02\left(\right.$ years $\left.^{-1}\right)$ \\
$\beta_{1}$ & \left.${\text { variable }\left(\text { years }^{-1}\right)}^{-1}\right)$ \\
$\beta_{2}$ & ${\text { variable }\left(\text { years }^{-1}\right)}_{\gamma_{0}}$ \\
$\gamma_{1}$ & $0.2\left(\right.$ years $\left.^{-1}\right)$ \\
$\gamma_{2}$ & $0.1\left(\right.$ years $\left.^{-1}\right)$ \\
$\mu_{0}$ & $0.08\left(\right.$ years $\left.^{-1}\right)$ \\
$a_{1}$ & $0.015\left(\right.$ years $\left.^{-1}\right)$ \\
$a_{2}$ & $0.03\left(\right.$ years $\left.^{-1}\right)$ \\
$\tau_{1}$ & $0.025\left(\right.$ years $\left.^{-1}\right)$ \\
$\tau_{2}$ & $0.2\left(\right.$ years $\left.^{-1}\right)$ \\
\hline
\end{tabular}

can be seen in Figure 2 that $R_{1}^{t h r}$ increases with increasing $\beta_{1}$ and a greater reduction in recidivism prevalence is recorded for decreasing values of $\beta_{2}$.

As stated earlier, the physical significance of the phenomenon of backward bifurcation is that the classical requirement of $R_{1}^{*}<1$ is no longer sufficient for avoiding the 


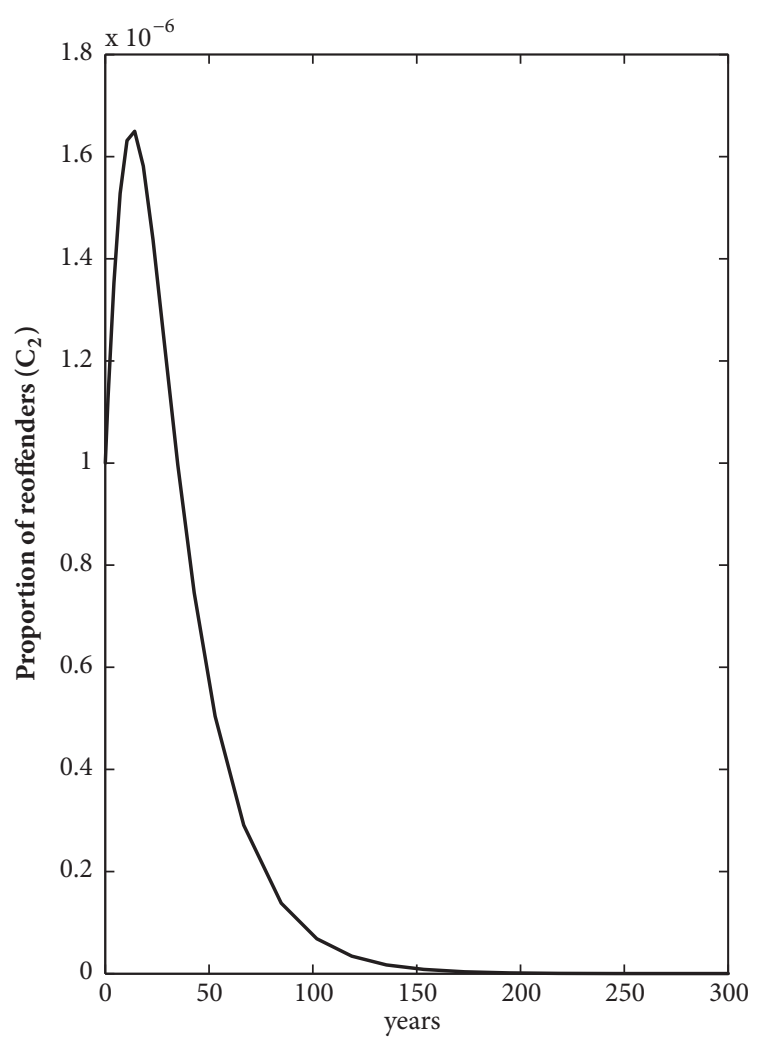

(a)

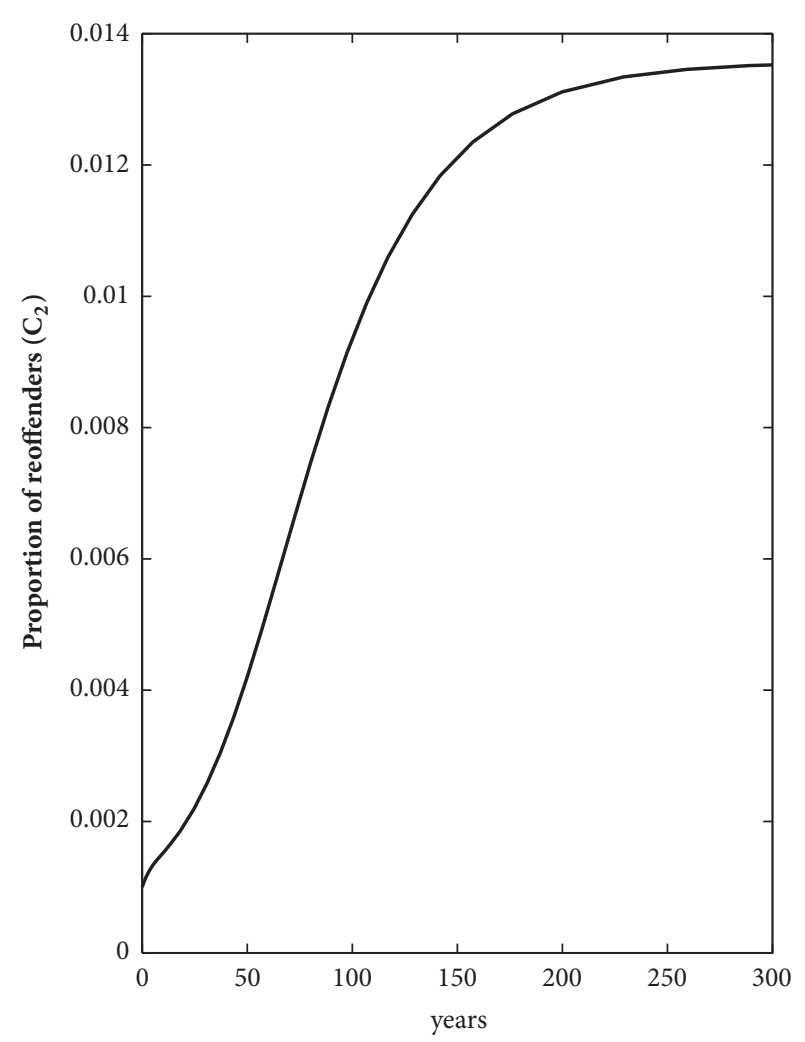

(b)

FIGURE 3: For $\beta_{1}=11, \beta_{2}=50$, and $R_{1}^{\text {thr }}<R_{1}^{*}<1$. The other parameters' values are given in Table 3. Profile of the population of reoffenders $\left(C_{2}\right)$. (a) If the recidivism is low (or $C_{2}(0)$ small), then the equilibrium point $P_{l}$ is locally asymptotically stable; (b) if the recidivism is higher (or $C_{2}(0)$ large), then the equilibrium point $P_{h}$ is locally asymptotically stable.

recidivism prevalence, as it is for the forward bifurcation. In such a scenario, the recidivism would depend on the initial sizes of the subpopulation $C_{2}$ of the model. That is, the presence of backward bifurcation in model (1) suggests that the possibility of avoiding the recidivism event when $R_{1}^{*}<1$ could be dependent on the initial sizes of the subpopulation $C_{2}$. This scenario is illustrated numerically in Figure 3. Thus, if the recidivism is low, then $C_{2}(0)$ is small, such that the low-criminality equilibrium $P_{l}$ is stable (Figure 3(a)). In contrast, if the recidivism is higher, then $C_{2}(0)$ is large, so the high-criminality equilibrium $P_{h}$ is stable (Figure 3(b)). Therefore, the stability of these equilibrium points depends on the initial condition of system (1). This clearly indicates the coexistence of two locally asymptotically stable equilibria when $R_{1}^{*}<1$, confirming that model (1) undergoes the phenomenon of backward bifurcation with one stable highcriminality equilibrium $P_{h}^{+}$(higher, solid curve in Figure 2), one unstable high-criminality equilibrium $P_{h}^{-}$(lowest dashed curve in Figure 2), and one low-criminality equilibrium $P_{l}$.

2.2.2. Analytic Strategy: $\beta_{1}$ Fixed with $\beta_{2}$ Increasing. Alternatively, from now on we explore the implications of the parameter $\beta_{2}$ on the criminal dynamics. It is worth mentioning that, from expression (10), it is easy to verify that $b_{1}<0$ if and only if

$$
\beta_{2}>\frac{\beta_{1}\left(\mu+\gamma_{2}\right)}{\left(\mu+\gamma_{1}\right)\left[R_{1}^{*}-\left(\mu+a_{2}\right) /\left(\tau_{2}+\mu+a_{2}\right)\right]}>0
$$

and thus, in such case, system (1) has two positive highcriminality equilibria (case (iii), see Theorem 1.) for $b_{0}>0$, $b_{1}^{2}-4 b_{2} b_{0}>0$, and $R_{1}^{*}>\left(\mu+a_{2}\right) /\left(\tau_{2}+\mu+a_{2}\right)$.

A schematic diagram of this bifurcation phenomenon for system (1) is given in Figure 4, where $\beta_{2}$ is chosen as a bifurcation parameter. As mentioned previously, this bifurcation phenomenon of model (1) is only illustrated numerically.

Figure 4 shows the profile of the proportion of both reoffenders $C_{2}^{+}$(solid curve) and $C_{2}^{-}$(dashed curve) as a function of $\beta_{2}$ with decreasing values of $\beta_{1}=30 ; 15.449 ; 11 ; 7.5 ; 5.845\left(\right.$ year $\left.^{-1}\right)$ as $\beta_{2}$ increases. The solid curve stands for the stable equilibrium and the dashed curve stands for the unstable equilibrium. Note that, for $\beta_{1}=30$ and $R_{1}^{*}>1, \beta_{2}=0$ such that system (1) has a unique positive high-criminality equilibrium (see case (i) of Theorem 1 and (12)).

It should be also noticed in Figure 4 that $R_{1}^{*}$ increases with decreasing $\beta_{2}$, such that greater reduction in recidivism prevalence is recorded for decreasing values of $\beta_{1}$. One aspect to differentiate both parameters $\beta_{1}$ and $\beta_{2}$ is the way that they account for the transition to criminal activity. 


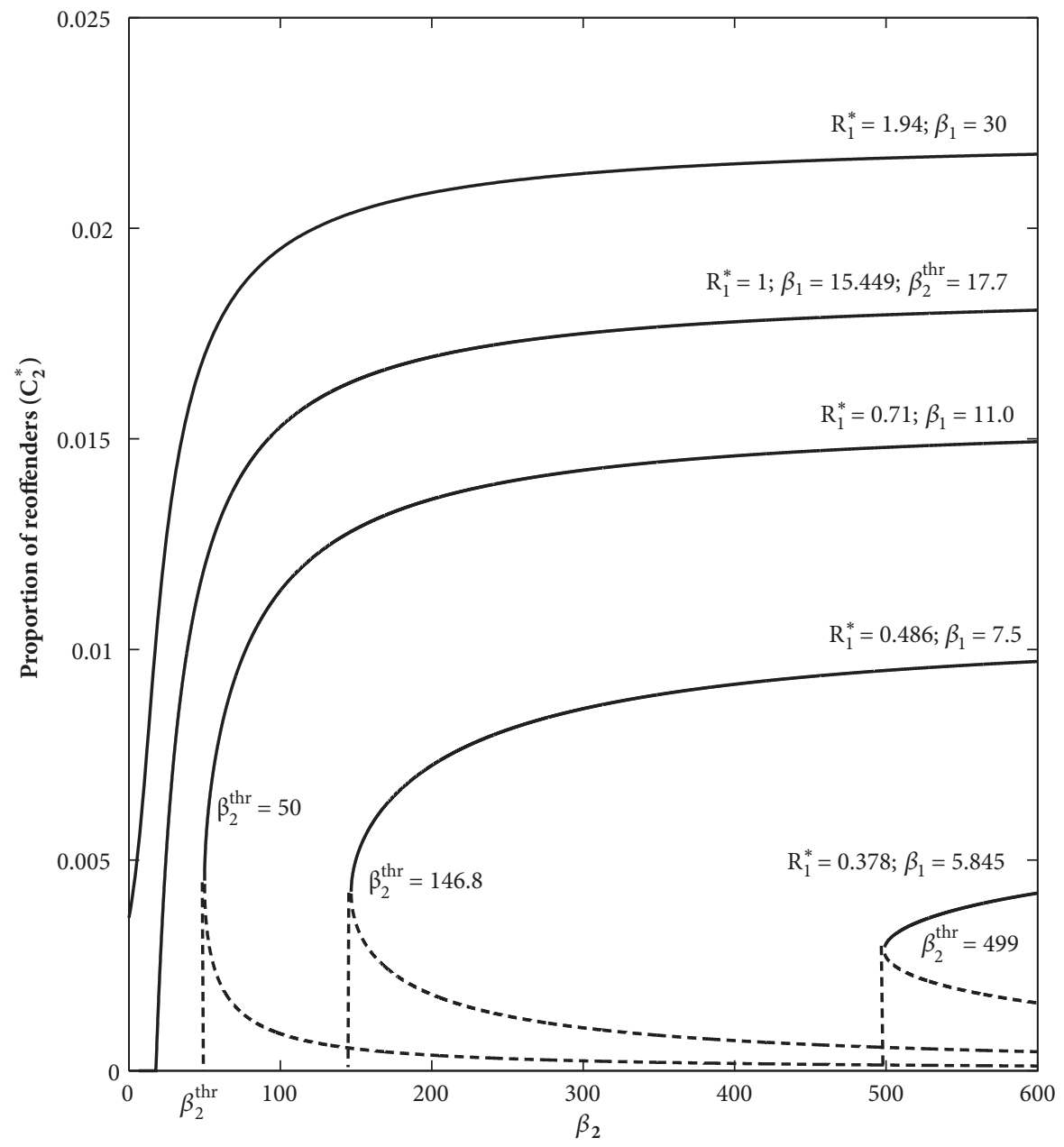

FiguRE 4: Breaking point and forward bifurcation diagrams for proportion of reoffenders $\left(C_{2}^{*}\right)$ for model (1), where $\beta_{2}$ is chosen as a bifurcation parameter ( $\beta_{1}$ fixed with $\beta_{2}$ increasing). The higher solution corresponds to the stable equilibrium (solid curve); the smaller solution corresponds to the unstable equilibrium (dashed curve). The phenomenon of the breaking point for (a) $\beta_{1}=11, R_{1}^{*}=0.71$, and $\beta_{2}^{\text {thr }}=50$; (b) $\beta_{1}=7.5, R_{1}^{*}=0.486$, and $\beta_{2}^{\text {thr }}=146.8$; and (c) $\beta_{1}=5.845, R_{1}^{*}=0.378$, and $\beta_{2}^{\text {thr }}=499$; forward bifurcation for $\beta_{1}=15.449$, $R_{1}^{*}=1$, and $\beta_{2}^{\text {thr }}=17.7$. For $\beta_{1}=30$ and $R_{1}^{*}=1.94, \beta_{2}=0$ such that system (1) has a unique positive high-criminality equilibrium $P_{h}$. Parameters' values used are as given in Table 3 .

In the low-criminality scenario, the only way someone becomes criminally active is on his/her own, without interacting with another person who is already criminally active. This restriction is necessary in order to illustrate the dynamics of a system which would lead to low-criminality equilibrium. If new criminal could only emerge on his/her own, then reducing the number of individuals who are not criminally active (core group) through either intervention or spontaneous desistance could lead to the lowest criminal scenario. For this, we calculated the threshold between the low-criminality equilibrium and the high-criminality equilibrium. Therefore, for $R_{1}^{*}<R_{1}^{\text {thr }}$, system (1) presented the locally asymptotically stable low-criminality equilibrium $P_{l}$. Although not a realistic possibility, understanding the intrinsic criminal propensity that leads to such kind of deviant behavior is a necessary step for understanding the high-criminality prevalence in the model, which will be presented in the next section.
In addition, in the high-criminality scenario, the way someone can become criminally active is to interact with another person who is already criminally active. Thus, if new reoffenders could only emerge by interaction between reoffenders $\left(C_{2}^{*}\right)$ and those second-time offenders susceptible to the crime $\left(S_{2}^{*}\right)$ at a rate $\beta_{2}$, then reducing the number of contacts between $C_{2}^{*}$ and $S_{1}$, that is, reducing $\beta_{1}$, could lead to the low-criminality scenario if the proportion of reoffenders $C_{2}^{*}$ is small (see Figure 3 ). Hence, to reach the low-criminality equilibrium, a community needs to reduce $\beta_{1}$. However, if $\beta_{1}$ is large, then the proportion of reoffenders $C_{2}^{*}$, as well as $S_{2}^{*}$, increases, which leads to higher crime prevalence, such that the high-criminality scenario emerges.

Figure 5(a) shows the long-term behavior of $\ln \beta_{2}^{\text {thr }}$ plotted versus $\beta_{1}$. The threshold $\beta_{2}^{\text {thr }}$ is expressed as a minimum value where system (1) has two positive equilibrium points. Thus, for each fixed $\beta_{1}$, there is a corresponding unique 

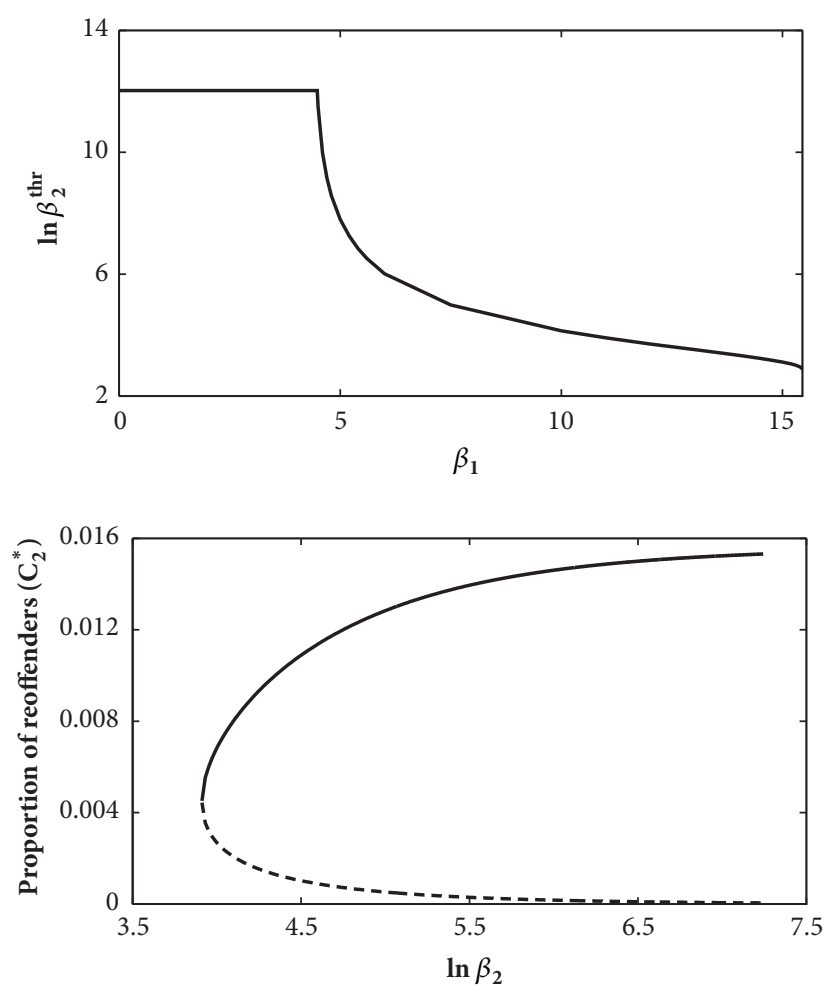

FIgURE 5: (a) The long-term behavior of $\ln \beta_{2}^{\text {thr }}$ plotted versus $\beta_{1}$. (b) For $\beta_{1}=11, R_{1}^{*}=0.71$, and $\ln \beta_{2}=3.91$, system (1) has two positive equilibrium points. The solid curve stands for the stable equilibrium and the dashed curve stands for the unstable equilibrium. The other parameters' values are given in Table 3 .

threshold $\beta_{2}^{\text {thr }}$. For one such example (see Figure 5(b)), $\beta_{1}=$ $11, R_{1}^{*}=0.71$, and $\beta_{2}^{\text {thr }}=50$ (or $\ln 50=3.91$ ) are the minimum values where system (1) has two positive value. For $\beta_{2}>\beta_{2}^{\text {thr }}$, we have $C_{2}^{+}>0$ and $C_{2}^{-}>0$. In this case, system (1) has a stable equilibrium point, $C_{2}^{+}$(the solid curve in Figure 5(b)), and an unstable equilibrium point, $C_{2}^{-}$(the dashed curve in Figure 5(b)). On the other hand, for $\beta_{2}<$ $\beta_{2}^{\text {thr }}$, we have $C_{2}^{+}=C_{2}^{-}=0$, which means that there are no positive equilibrium points for system (1). Moreover, for $\beta_{1}^{\text {thr }}=15.4492917$, we have $R_{1}^{*}=1$ and $\beta_{2}^{\text {thr }}=17.867$ (or $\ln 17.867=2.883$ ) such that, for $\beta_{2}<\beta_{2}^{\text {thr }}$, one has $C_{2}^{+}=0$ and $C_{2}^{-}<0$. Otherwise, for $\beta_{2}>\beta_{2}^{\text {thr }}$, one has $C_{2}^{+}>0$ and $C_{2}^{-}=0$ (see Figure 6).

\subsection{The Full Contagion Criminal Model (FCCM) Formulation.} In this section, we relax the assumption made in the PCCM model (1) that the susceptible individuals $\left(S_{0}\right)$ can enter to crime only on their own, so we make $\delta_{0}=0$. Hence, in the modified model, the flow of the susceptible individuals $S_{0}$ into the criminal activity depends only on the contact with those individuals who are incarcerated, $C_{1}$. We also define $\beta_{i}(\mathrm{i}=$ $0,1,2)$ as the rate of imprisonment; it captures the return to criminal activity of those released from prison, such that $\beta_{0}$, $\beta_{1}$, and $\beta_{2}$ are the flows into criminal activity which depend on having had contact with those first-time and second-time incarcerated individuals $\left(C_{1}\right.$ and $\left.C_{2}\right)$, respectively. Following the idea of the previous model $(1)$, here $\beta_{i}(i=0,1,2)$ is analogous to the effective contact rate in infectious diseases model. This derivation adopts a standard incidence formulation, where the contact rate is assumed to be constant, unlike the case of the mass action formulation, where the contact rate depends on the size of the total population (see, e.g., [27, 30], for detailed derivation of these incidences' functions).

The flow diagram of the FCCM is depicted in Figure 7. The variables and parameters' values are given in Table 3, except for $\beta_{0}$ that is the contact rate between $C_{1}$ and $S_{0}$ (cooptation rate).

The model is represented by the following nonlinear system of differential equations:

$$
\begin{aligned}
& \frac{d S_{0}}{d t}=\Lambda-\beta_{0} C_{1} S_{0}-\left(\gamma_{0}+\mu\right) S_{0} \\
& \frac{d D_{0}}{d t}=\gamma_{0} S_{0}-\mu D_{0} \\
& \frac{d C_{1}}{d t}=\beta_{0} C_{1} S_{0}-\left(\tau_{1}+\mu+a_{1}\right) C_{1} \\
& \frac{d S_{1}}{d t}=\tau_{1} C_{1}-\left(\gamma_{1}+\mu\right) S_{1}-\beta_{1} C_{2} S_{1} \\
& \frac{d D_{1}}{d t}=\gamma_{1} S_{1}-\mu D_{1} \\
& \frac{d C_{2}}{d t}=\left(\beta_{1} S_{1}+\beta_{2} S_{2}\right) C_{2}-\left(\tau_{2}+\mu+a_{2}\right) C_{2} \\
& \frac{d S_{2}}{d t}=\tau_{2} C_{2}-\beta_{2} C_{2} S_{2}-\left(\gamma_{2}+\mu\right) S_{2} \\
& \frac{d D_{2}}{d t}=\gamma_{2} S_{2}-\mu D_{2}
\end{aligned}
$$

\subsection{The Existence and Local Stability of Equilibria}

2.4.1. Crime-Free Equilibrium. In the absence of crime, that is, for $C_{1}=C_{2}=0$, model (15) has a crime-free equilibrium $P_{0}=\left(S_{0}^{0}, D_{0}^{0}, 0,0,0,0,0,0\right)$ which is obtained by setting the right-hand sides of system (15) to zero, where

$$
\begin{aligned}
S_{0}^{0} & =\frac{\Lambda}{\left(\mu+\gamma_{0}\right)}, \\
D_{0} & =\frac{\gamma_{0}}{\mu} S_{0}^{0} .
\end{aligned}
$$

To analyze the local stability of this equilibrium, the Jacobian of system (15) is computed and evaluated at $P_{0}$, which is locally asymptotically stable if the real parts of the eigenvalues of the Jacobian matrix are all negative.

Thus, the local stability of the crime-free equilibrium $P_{0}$ is governed by the Jacobian matrix

$$
M_{P_{0}}=\left[\begin{array}{cc}
A_{P_{0}} & 0 \\
0 & B_{P_{0}}
\end{array}\right] \text {, }
$$




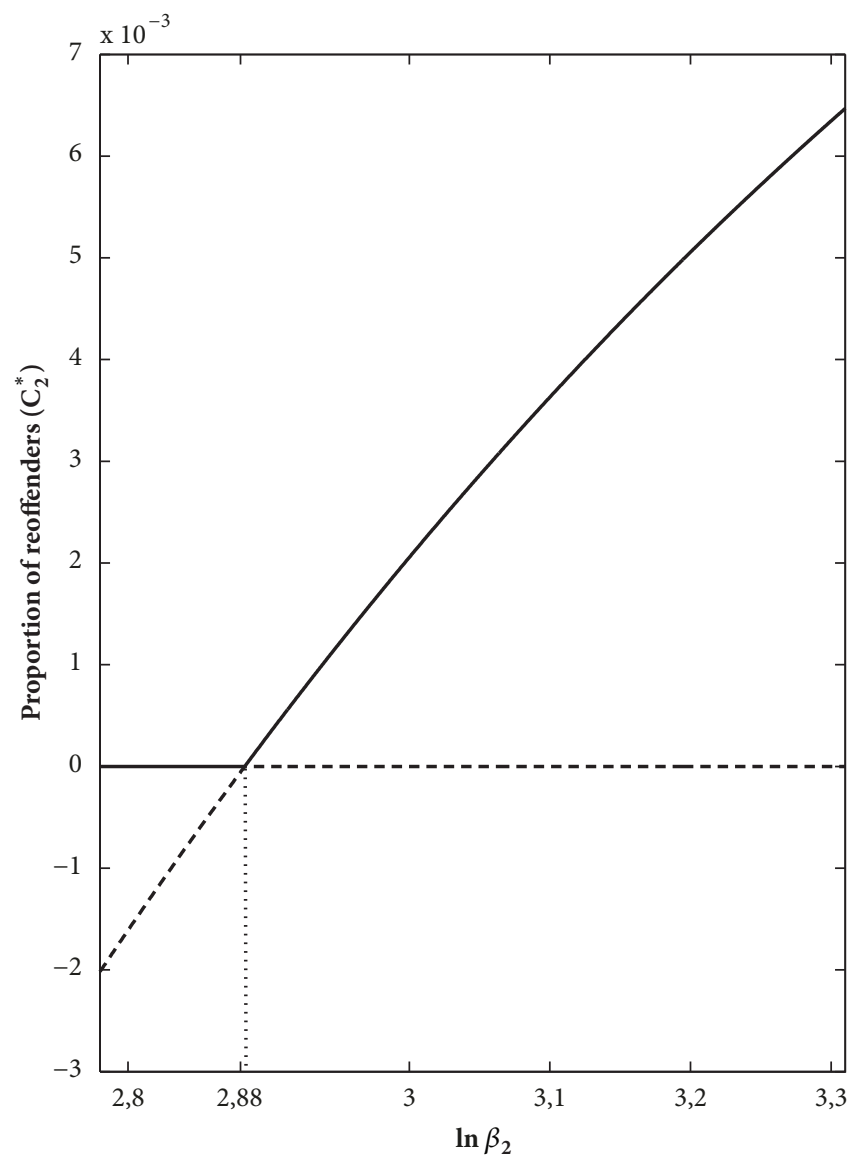

FIGURE 6: Long-term physical outcome $C_{2}^{*}$ plotted versus $\ln \beta_{2}$. For $\beta_{1}^{\text {thr }}=15.449, R_{1}^{*}=1$, and $\beta_{2}>\beta_{2}^{\text {thr }}$, system (1) has one positive equilibrium point. The solid curve stands for the stable equilibrium and the dashed curve stands for the unstable equilibrium. The other parameters' values are given in Table 3.

where

$$
\begin{aligned}
A_{P_{0}} & =\left[\begin{array}{cc}
-\left(\mu+\gamma_{0}\right) & -\beta_{0} S_{0}^{0} \\
0 & \beta_{0} S_{0}^{0}-\left(\tau_{1}+\mu+a_{1}\right)
\end{array}\right], \\
B_{P_{0}} & =\left[\begin{array}{ccc}
-\left(\mu+\gamma_{1}\right) & 0 & 0 \\
0 & -\left(\tau_{2}+\mu+a_{2}\right) & 0 \\
0 & \tau_{2} & -\left(\mu+\gamma_{2}\right)
\end{array}\right] .
\end{aligned}
$$

It is easy to verify that the three eigenvalues of matrix (19) are always negative. In the same way, it is also straightforward to verify that one of the eigenvalues of matrix (18) is always negative, while the other is negative whenever

$$
R_{0}=\frac{\beta_{0}}{\left(\tau_{1}+\mu+a_{1}\right)} S_{0}^{0}<1 .
$$

Hence, all the eigenvalues of matrix (17) are negative or have negative real parts if and only if $R_{0}<1$. In summary, the crime-free equilibrium $P_{0}$ of system (15) is locally asymptotically stable if the Basic Criminality Reproduction Number $R_{0}<1$. Therefore, we have established the following result.

Lemma 2. The crime-free equilibrium $P_{0}$ of system (15) is locally asymptotically stable if $R_{0}<1$ and is unstable if $R_{0}>1$.
2.4.2. Low-Criminality Equilibrium. In the absence of the reoffenders, that is, for $C_{2}=0$, model (15) has a lowcriminality equilibrium $P_{l}^{*}=\left(S_{0}, D_{0}, C_{1}, S_{1}, D_{1}, 0,0,0\right)$, where

$$
\begin{aligned}
S_{0} & =\frac{\left(\tau_{1}+\mu+a_{1}\right)}{\beta_{0}} \\
D_{0} & =\frac{\gamma_{0}}{\mu} S_{0} \\
C_{1} & =\frac{\Lambda \beta_{0}-\left(\mu+\gamma_{0}\right)\left(\tau_{1}+\mu+a_{1}\right)}{\left(\tau_{1}+\mu+a_{1}\right) \beta_{0}} \\
& =\frac{\left(\mu+\gamma_{0}\right)}{\beta_{0}}\left(R_{0}-1\right) \\
S_{1} & =\frac{\tau_{1} C_{1}}{\left(\mu+\gamma_{1}\right)} \\
D_{1} & =\frac{\gamma_{1}}{\mu} S_{1} .
\end{aligned}
$$

Note that $P_{l}^{*}$ exists if $C_{1}>0$, that is, if $R_{0}>1$. Now consider the resulting model (15). The local stability 


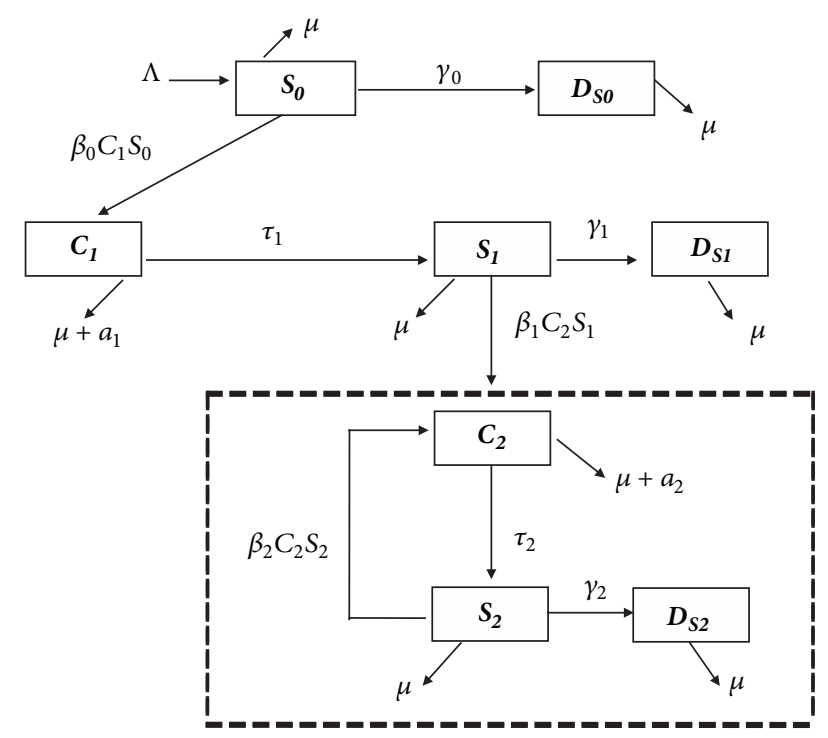

Figure 7: The flow diagram for the free-low-high criminality model (15).

of the low-criminality equilibrium $P_{l}$, which is examined by linearizing system (15) around $P_{l}$, is governed by the Jacobian matrix

$$
M_{P_{l}}=\left[\begin{array}{ccccc}
M_{11} & -\beta_{0} S_{0} & 0 & 0 & 0 \\
\beta_{0} C_{1} & M_{22} & 0 & 0 & 0 \\
0 & \tau_{1} & M_{33} & -\beta_{1} S_{1} & 0 \\
0 & 0 & 0 & M_{44} & 0 \\
0 & 0 & 0 & \tau_{2} & M_{55}
\end{array}\right],
$$

with $M_{11}=-\beta_{0} C_{1}-\left(\mu+\gamma_{0}\right) ; M_{22}=\beta_{0} S_{0}-\left(\tau_{1}+\mu+a_{1}\right)$; $M_{33}=-\left(\mu+\gamma_{1}\right) ; M_{44}=\beta_{1} S_{1}-\left(\tau_{2}+\mu+a_{2}\right)$; and $M_{55}=-\left(\mu+\gamma_{2}\right)$.

The eigenvalues of matrix (22) are $\lambda_{1}=M_{55}, \lambda_{2}=M_{44}$, $\lambda_{3}=M_{33}$, and the roots of

$$
\phi^{2}+z_{1} \phi+z_{0}=0
$$

with $z_{1}=\Lambda \beta_{0} /\left(\tau_{1}+\mu+a_{1}\right)>0$ and $z_{0}=\Lambda \beta_{0}-\left(\mu+\gamma_{0}\right)\left(\tau_{1}+\right.$ $\left.\mu+a_{1}\right)>0\left(\Longleftrightarrow R_{0}>1\right)$.

The eigenvalues $\lambda_{1}$ and $\lambda_{3}$ are real negative from definition of the parameters $\mu, \gamma_{1}$, and $\gamma_{2}$. The eigenvalue $\lambda_{2}$ is negative if

$$
\beta_{1}<\frac{\left(\tau_{2}+\mu+a_{2}\right)}{S_{1}}=\frac{\beta_{0}\left(\tau_{2}+\mu+a_{2}\right)\left(\mu+\gamma_{1}\right)}{\tau_{1}\left(\mu+\gamma_{0}\right)\left(R_{0}-1\right)}
$$

or, equivalently, if

$$
R_{1}=\frac{\beta_{1} \tau_{1}\left(\mu+\gamma_{0}\right)\left(R_{0}-1\right)}{\left(\tau_{2}+\mu+a_{2}\right)\left(\mu+\gamma_{1}\right) \beta_{0}}<1
$$

Finally, the eigenvalues $\lambda_{4}$ and $\lambda_{5}$ are real negative if $z_{0}>$ 0 and $z_{1}>0$ by applying the Routh-Hurwitz criteria $[31,32]$ on polynomial (23). In this sense, $z_{0}>0$ and $z_{1}>0$ if and only if $R_{0}>1$.

Thus, we have established the following result.
Lemma 3. The low-criminality equilibrium $P_{l}^{*}$ of system (15) exists and it is locally asymptotically stable if $R_{0}>1$ and $R_{1}<$ 1. Otherwise, $P_{l}^{*}$ is unstable.

It should be mentioned that the consequence of the above result is that when $P_{l}^{*}$ becomes unstable, two scenarios emerge: one for the case where the criminality is eliminated from population and $P_{0}$ is stable and the other where new criminals could emerge and there is high-criminality prevalence in the population such that model (15) has a highcriminality equilibrium with coexistence of both offenders: $C_{1} \neq 0$ and $C_{2} \neq 0$. In this way, it is instructive to determine the possible interventions on $\beta_{0}$ and $\beta_{1}$ in order to reduce $R_{0}$ and $R_{1}$ below one, that is, to guarantee the conditions under which the criminality is eliminated, or at least having its incidence reduced.

2.4.3. High-Criminality Equilibrium. In what follows, if $R_{1}>$ 1 , such that the low-criminality equilibrium $P_{l}^{*}$ is unstable, system (15) has the positive high-criminality equilibrium, $P_{h}^{*}=\left(S_{0}, D_{0}, C_{1}, S_{1}^{*}, D_{1}^{*}, C_{2}^{*}, S_{2}^{*}, D_{2}^{*}\right)$, where

$$
\begin{aligned}
& S_{1}^{*}=\frac{\tau_{1} C_{1}}{\mu+\gamma_{1}+\beta_{1} C_{2}^{*}}, \\
& S_{2}^{*}=\frac{\tau_{2} C_{2}^{*}}{\mu+\gamma_{2}+\beta_{2} C_{2}^{*}}, \\
& D_{1}^{*}=\frac{\gamma_{1}}{\mu} S_{1}^{*}, \\
& D_{2}^{*}=\frac{\gamma_{2}}{\mu} S_{2}^{*} .
\end{aligned}
$$

Remembering from (21) that $C_{1}>0 \Longleftrightarrow R_{0}>1$, so $P_{h}^{*}$ exists if and only if $R_{0}>1$. Now replacing the expressions for $S_{1}^{*}$ and $S_{2}^{*}$ in the sixth equation of system (15), the positive high-criminality equilibrium, which cannot be expressed cleanly in closed form, can then be obtained by solving for $C_{2}^{*}$ the following expression:

$$
\eta_{2}\left(C_{2}^{*}\right)^{2}+\eta_{1} C_{2}^{*}+\eta_{0}=0
$$

where

$$
\begin{aligned}
\eta_{2} & =\beta_{1} \beta_{2}\left(\mu+a_{2}\right), \\
\eta_{1} & =\left(\mu+\gamma_{1}\right)\left(\tau_{2}+\mu+a_{2}\right) \\
& \cdot \beta_{2}\left\{\left[\frac{\beta_{1}\left(\mu+\gamma_{2}\right)}{\beta_{2}\left(\mu+\gamma_{1}\right)}+\frac{\left(\mu+a_{2}\right)}{\left(\tau_{2}+\mu+a_{2}\right)}\right]-R_{1}\right\} \\
\eta_{0} & =\left(\mu+\gamma_{1}\right)\left(\mu+\gamma_{2}\right)\left(\mu+\gamma_{2}+a_{2}\right)\left(1-R_{1}\right) .
\end{aligned}
$$

Since all the model's parameters are nonnegative, it follows from (28) that the coefficient $\eta_{2}$ is always positive and $\eta_{0}<0$ for $R_{1}>1$. Thus, it is clear that model (15) has a unique positive equilibrium $P_{h}^{+}$when $R_{1}>1$ and $R_{0}>1$. For $\eta_{0}=0$ and $\eta_{1}<0$, model (15) also has a unique positive equilibrium 


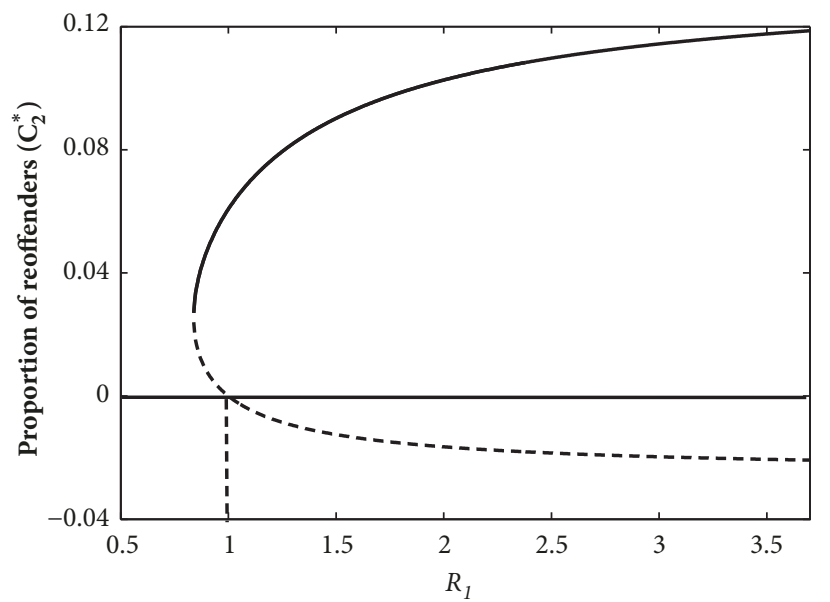

(a)

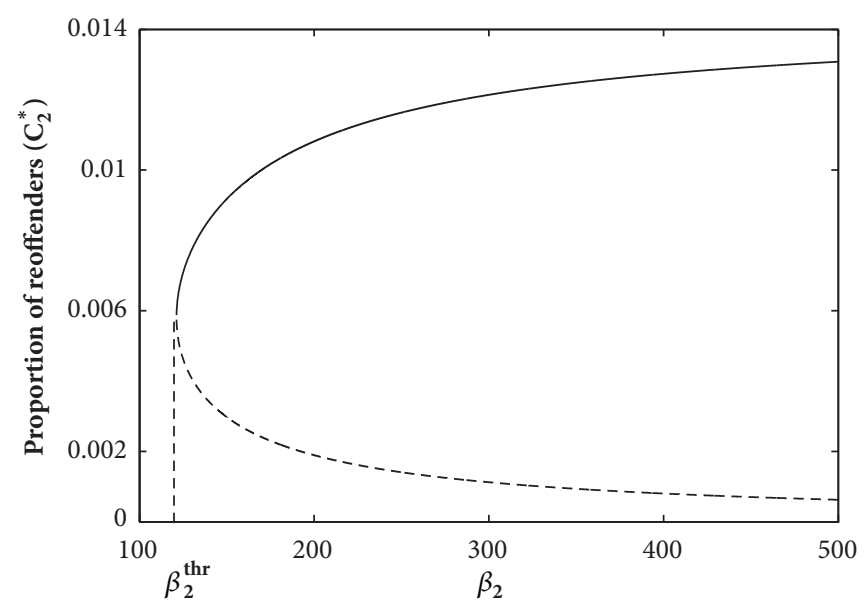

(b)

FIGURE 8: The phenomena of backward bifurcation and breaking point, when $\beta_{1}$ and $\beta_{2}$ are chosen as a bifurcation parameter. (a) Backward bifurcation for $\beta_{2}=5$ with $\beta_{0}$ and $\beta_{1}$ increasing. (b) Breaking point for $\beta_{0}=\beta_{1}=5$ with $\beta_{2}$ increasing, where $\beta_{2}^{\text {thr }}=121.2$ and $R_{0}=1.139$ and $R_{1}=0.464$. The higher solution corresponds to the stable equilibrium (solid curve); the smaller solution corresponds to the unstable equilibrium (dashed curve). Parameters' values used are as given in Table 3.

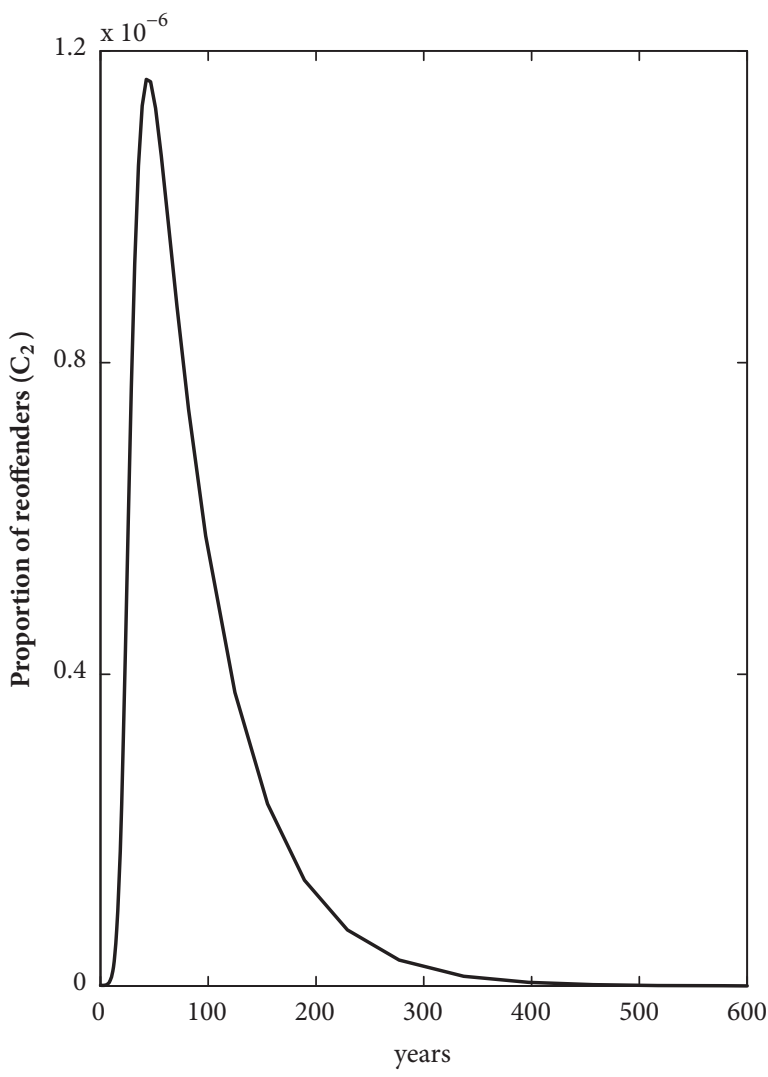

(a)

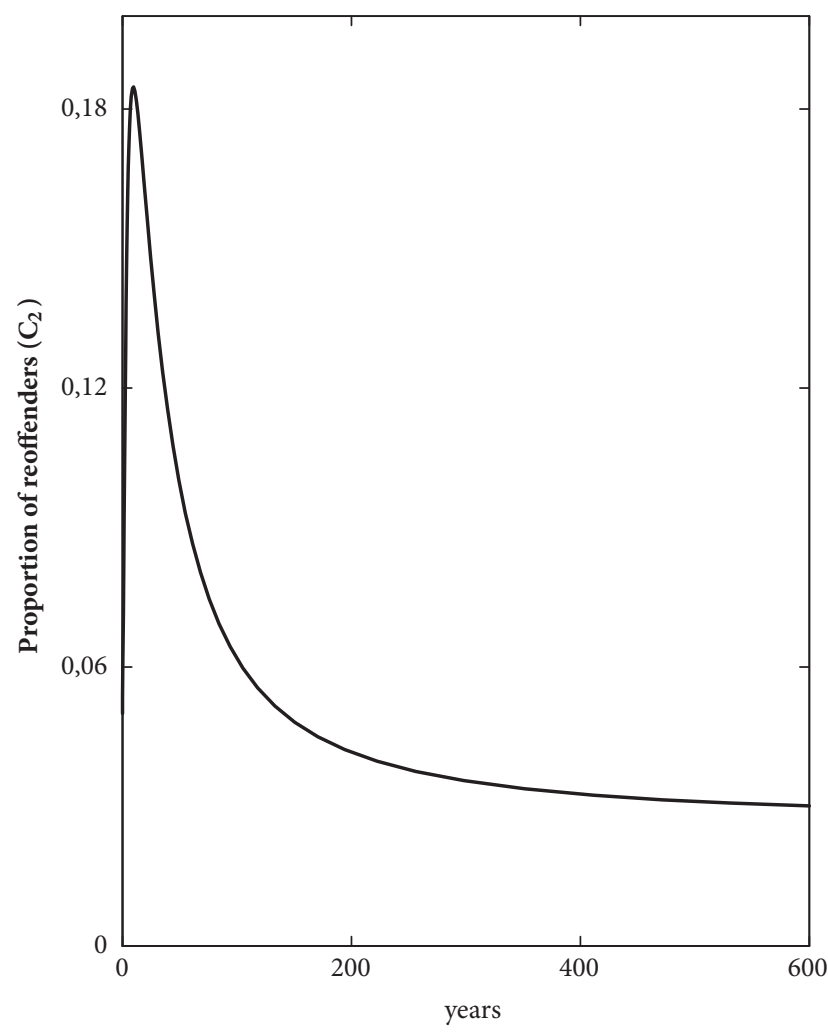

(b)

FIGURE 9: For $\beta_{1}=2.0, \beta_{2}=4.4\left(\beta_{2}>\beta_{2}^{\text {thr }}\right), R_{0}=2.39$, and $R_{1}=0.89$. The other parameters' values are given in Table 3. Profile of population of reoffenders $\left(C_{2}\right)$. (a) If the recidivism is low (or $C_{2}(0)$ small), then the equilibrium point $P_{l}^{*}$ is locally asymptotically stable. (b) If the recidivism is higher (or $C_{2}(0)$ large), then the equilibrium point $P_{h}^{+}$is locally asymptotically stable. 


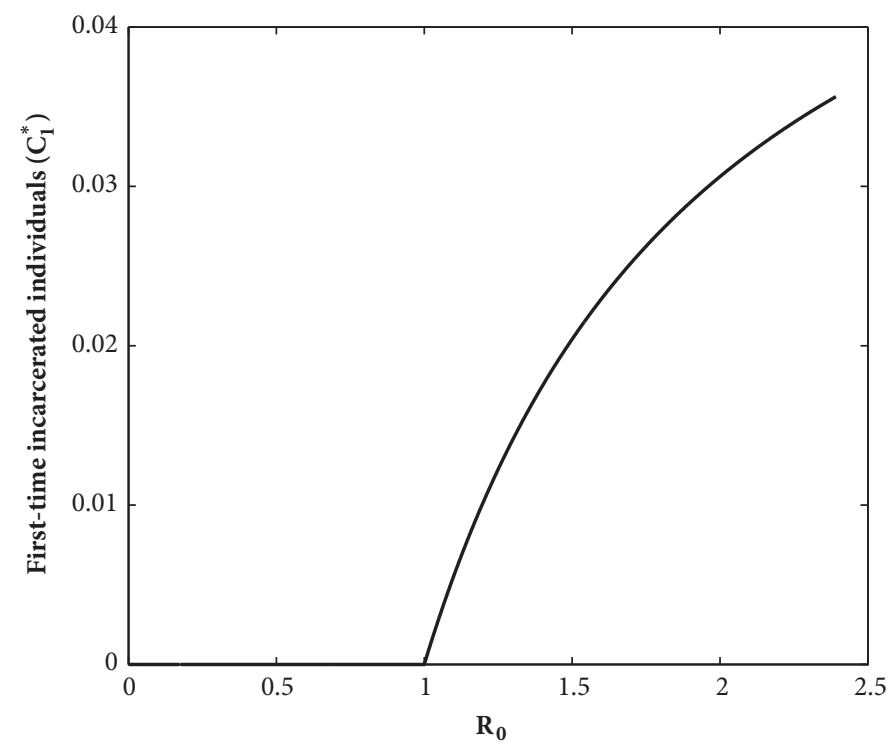

FIGURE 10: The phenomenon of forward bifurcation when $\beta_{0}$ is chosen as a bifurcation parameter. The crime-free equilibrium $P_{0}$ of system (15) is locally asymptotically stable if $R_{0}<1$ and is unstable if $R_{0}>1$. Parameters' values used are as given in Table 3 .

$P_{h}^{+}$. Moreover, whenever $R_{0}>1$, if $R_{1}=1$, then $\eta_{0}=0$ and $\eta_{1}<0$ if only if

$$
\beta_{2}>\frac{\left(\tau_{2}+\mu+a_{2}\right)\left(\mu+\gamma_{2}\right) \beta_{1}}{\tau_{2}\left(\mu+\gamma_{1}\right)}
$$

such that model (15) also has a unique positive equilibrium $P_{h}^{*}$. Finally, for $\eta_{0}>0, \eta_{1}<0$, and $\eta_{1}^{2}-4 \eta_{2} \eta_{0}>0$, that is, for $R_{0}>1$ and $R_{1}<1$, model (15) has two positive equilibria $P_{h}^{+}$ and $P_{h}^{-}$. Note that these equilibrium points cannot be studied in its closed form, so we carried out its local stability using numerical methods.

Thus, the following result is then established.

Theorem 4. Model (15) has

(i) a unique positive equilibrium $P_{h}^{+}$if $\eta_{0}<0 \Longleftrightarrow R_{1}>$ 1 ;

(ii) a unique positive equilibrium $P_{h}^{+}$if $\eta_{0}=0$ and $\eta_{1}<0$;

(iii) two positive equilibria, $P_{h}^{+}$and $P_{h}^{-}$, if $\eta_{0}>0$ and $\eta_{1}<0$ and $\eta_{1}^{2}-4 \eta_{2} \eta_{0}>0$

(iv) no positive equilibrium, otherwise.

As explored in model (1), model (15) also exhibits the phenomenon of backward bifurcation (Figure 8(a)) and breaking point (Figure $8(\mathrm{~b})$ ) when $\beta_{1}$ and $\beta_{2}$ are chosen as bifurcation parameters.

Figure 8(a) shows the backward bifurcation for $\beta_{2}=5$ and $\beta_{0}=8.4\left(R_{0}=2.39\right)$ with $\beta_{1}$ increasing. Figure $8(\mathrm{~b})$ shows the phenomenon of breaking point for $\beta_{1}=\beta_{0}=5$, with $\beta_{2}$ increasing, where $\beta_{2}^{*}=121.2$ and $R_{0}=1.139$ and $R_{1}=0.464$. As it should be expected, Figures 8(a) and 8(b) present the same results shown in Figures 2 and 4. In summary, if $R_{0}>1$ and $R_{1}>1$, model (15) has the locally asymptotically stable high-criminality equilibrium $P_{h}^{+}$.
Finally, if $R_{0}>1$ and $R_{1}<1$ and $\beta_{2}>\beta_{2}^{\text {thr }}$, then there is coexistence of two locally asymptotically stable equilibria, $P_{l}^{*}$ and $P_{h}^{+}$, such that the stability of these equilibrium points depends on the initial condition of system (15) (see Figure 9).

In contrast to model (1), when $\beta_{0}$ is chosen as a bifurcation parameter, model (15) has the crime-free equilibrium $P_{0}$ given by (16), which indicates the possibility of the forward bifurcation (Figure 10). If $R_{0}<1$, then the crimefree equilibrium $P_{0}$ of system (15) is locally asymptotically stable; if $R_{0}>1, P_{0}$ becomes unstable and $P_{l}^{*}$ is locally asymptotically stable if $R_{1}<1$. Thus, the substitution of the assumption that the susceptible individuals $\left(S_{0}\right)$ can enter to crime only on their own (i.e., $\delta_{0}$ ) by the standard incidence (i.e., $\beta_{1} C_{1} S_{0}$ ) in model (1) includes the forward bifurcation phenomenon to model (15).

\section{Results}

To illustrate the theoretical results contained in this paper, models (1) and (15) are simulated using baseline parameters values/ranges summarized in Table 3 (unless otherwise stated). The parameters are chosen for simulations purposes only, so we could illustrate our qualitative results. Moreover, it is worth mentioning that if the assumption made in the PCCM model (1), that is, susceptible individuals can enter to crime only on their own (i.e., $\delta_{0} \neq 0$ ), and the assumptions made in FCCM model (15), that is, susceptible individuals get into criminal behavior depending only on the contact with those individuals who are incarcerated, $C_{1}$ (i.e., $\beta_{0} C_{1} S_{0}$ ), were considered in a single model, this new model would be more realistic. However, the analytical results and the phenomena of backward bifurcation and breaking point of this new model would be similar to model (1), except for the fact that $R_{0}$ would no longer exist. For this reason, we did not study this more realistic mixed model. 


\section{Discussion}

In this paper, we attempt to present theoretical models of criminal careers using the dynamical system approach traditionally used in the study of infectious diseases spreading in a homogeneous population. The models consider crime dynamics as contagious phenomena in which a susceptible cohort of individuals with criminal propensity is "infected" by criminal individuals who have been convicted for their crimes and are arrested in a prison. The contagion occurs by several ways, in particular through the sending of messages by mobile phones (a very widespread habit in Brazilian prisons) and through "carrier pigeons" represented by relatives and lawyers.

We propose, to the best of our knowledge by the first time in quantitative criminology, the existence of thresholds for the stability of crime-endemic equilibrium which are the equivalent to the "basic reproduction number" widely used in the mathematical epidemiology literature [22], as shown in Lemmas 2 and 3. Both model (1) and model (15), however, exhibit the phenomena of backward bifurcation and breaking point when the contact rates $\beta_{1}$ and $\beta_{2}$ are chosen as bifurcation parameters. Since data strongly suggests that standard incidence formulation is more suited for modeling human diseases $[22,23,27]$ and we adopted this formulation in the current study, the above results show that the phenomena of the backward bifurcation and the breaking point could be important properties of the criminality model.

As in other criminal career models, our models seek to provide a theoretical framework to analyse the longitudinal behavior of individuals who commit criminal offenses [33]. In addition, we centrered our analysis, although in an implicit way, in the parameter considered to be the most important for the analysis of criminal career, namely, the rate at which offenders commit crime, denoted in the specialized literature by the Greek letter $\lambda$, coincidently, the same symbol used to denote the force-of-infection in epidemiology of transmissible diseases.

Other dynamical system models proposed the analysis of criminal dynamics including differential equations, like the works by Farrington synthesized in Refs. [16, 34]. However, these models are either linear (the former) or related to other kinds of infectious contagion (the latter).

Finally, it is noteworthy that the models proposed in the paper are intended only to provide a theoretical framework upon which other works can provide empirical support for the assumptions and values for the parameters determinant of the dynamical behavior of the systems here studied. The present work, therefore, is intended to provide the first step in the study of criminal careers as determined by contagious events like the ones related to the phenomenon of incarcerated criminals influencing the behavior of susceptible juveniles outside prisons with a criminal propensity. The finding of backward bifurcation in both models, however, implies that there is an endemic equilibrium of criminality even when the threshold parameter for contagion is below unit which, in turn, implies that control strategies are more difficult to achieve any considerable impact on crime control in situations similar to the ones here analyzed.

\section{Data Availability}

No data were used to support this study.

\section{Conflicts of Interest}

The authors declare that they have no conflicts of interest.

\section{Acknowledgments}

This work has been funded by LIM01-HCFMUSP, FAPESP, $\mathrm{CNPq}$ and by the Office of Naval Research, USA, Grant Number 2059558. An earlier version of this work has been presented as at EMAp Seminars according to the following link: https:/emap.fgv.br/seminarios/contagiouscriminal-career-models-showing-backward-bifurcationsimplications-crime.

\section{References}

[1] J. R. Kling and J. Ludwig, "Is Crime Contagious?" The Journal of Law and Economics, vol. 50, no. 3, pp. 491-518, 2007.

[2] C. F. Manski, "Identification of endogenous social effects: the reflection problem," Review of Economic Studies, vol. 60, no. 3, pp. 531-542, 1993.

[3] K. A. Goss and P. Cook A, A Selective Review of the SocialContagion Literature, Terry Sanford Instiute Working Paper, Duke University, Durham, NC, USA, 1996.

[4] C. F. Manski, "Economic analysis of social interactions," Journal of Economic Perspectives (JEP), vol. 14, no. 3, pp. 115-136, 2000.

[5] W. J. Wilson, The Truly Disadvantaged: The Inner City, the Underclass, and Public Policy, The University of Chicago Press, Chicago, Illinois, 1987.

[6] R. J. Sampson, S. W. Raudenbush, and F. Earls, "Neighborhoods and violent crime: a multilevel study of collective efficacy," Science, vol. 277, no. 5328, pp. 918-924, 1997.

[7] C. Jencks and S. E. Mayer, "The Social Consequences of Growing Up," in Inner-City Poverty in the United States, L. Lynn and M. McGeary, Eds., National Academy of Sciences, Washington, DC, USA, 1990.

[8] E. L. Glaeser, B. Sacerdote, and J. A. Scheinkman, "Crime and social interactions," The Quarterly Journal of Economics, vol. 111, no. 2, pp. 507-548, 1996.

[9] E. L. Glaeser, B. I. Sacerdote, and J. A. Scheinkman, "The social multiplier," Journal of the European Economic Association, vol. 1, no. 2-3, pp. 345-353, 2003.

[10] J. Crane, "The epidemic theory of ghettos and neighborhood effects on dropping out and teenage childbearing," American Journal of Sociology, vol. 96, no. 5, pp. 1226-1259, 1991.

[11] D. A. Levy and P. R. Nail, "Contagion: a theoretical and empirical review and reconceptualization," Genetic Social and General Psychology Monographs, vol. 119, no. 2, pp. 233-284, 1993.

[12] J. Fagan, D. L. Wilkinson, and G. Davies, "Social contagion of violence," in The Cambridge Handbook of Violent Behavior and Aggression, D. Flannery, A. T. Vazsonyi, and I. D. Waldman, Eds., Cambridge University Press, Cambridge, UK, 2007.

[13] R. S. Burt, "Social Contagion and Innovation: Cohesion versus Structural Equivalence," American Journal of Sociology, vol. 92, no. 6, pp. 1287-1335, 1987. 
[14] L. O. Gostin, "The interconnected epidemics of drug dependency and AIDS," Harvard Civil Rights Civil Liberties Law Review, vol. 26, no. 1, pp. 113-184, 1991.

[15] J. L. Rodgers and D. C. Rowe, "Social contagion and adolescent sexual behavior: A developmental EMOSA model," Psychological Review, vol. 100, no. 3, pp. 479-510, 1993.

[16] S. B. Patten, "Epidemics of violence," Medical Hypotheses, vol. 53, no. 3, pp. 217-220, 1999.

[17] E. Massad, A. F. Rocha, F. A. B. Coutinho, and L. F. Lopez, "Modelling the spread of memes: How inovations are transmitted from brain to brain," Applied Mathematical Sciences, vol. 7, no. 45-48, pp. 2295-2306, 2013.

[18] K. Kirkpatrick, "The Social Contagion of Violence: A Theoretical Exploration of the Nature of Violence in Society," in Social Science, vol. 461, 462, California Polytechnic State University, San Luis Obispo, CA, USA, 2018, http://digitalcommons .calpoly.edu/socssp/78.

[19] S. B. Patten and J. A. Arboleda-Flórez, "Epidemic theory and group violence," Social Psychiatry and Psychiatric Epidemiology, vol. 39, no. 11, pp. 853-856, 2004.

[20] C. C. N. Dias, PCC: Hegemonia nas prisões e monopólio da violência (Portuguese), Editora Saraiva, São Paulo, Brazil, 2013.

[21] S. Adorno and F. Salla, "Criminalidade organizada nas prisões e os ataques do PCC," Estudos Avançados, vol. 21, no. 61, pp. 7-29, 2007.

[22] R. M. Anderson and R. M. May, Population Biology of Infectious Diseases, Springer-Verlag, Berlin, Heidelberg, New York, 1982.

[23] R. M. Anderson and R. M. May, Infectious Diseases of Humans: Dynamics and Control, Oxford University Press, Oxford, England, 2nd edition, 1991.

[24] S. Machin, M. Olivier, and V. Suncica, "The crime reducing effect of education," Economic Journal, vol. 121, no. 552, pp. 463484, 2011.

[25] M. Maguire, "Merry Morash: Understanding Gender, Crime and Justice," Critical Criminology, vol. 16, no. 3, pp. 225-227, 2008.

[26] J. K. Hale, Ordinary Differential Equations, Krieger, Basel, Switzerland, 1980.

[27] H. W. Hethcote, "The mathematics of infectious diseases," SIAM Review, vol. 42, no. 4, pp. 599-653, 2000.

[28] V. Lakshmikantham, S. Leela, and A. A. Martynyuk, Stability Analysis of Nonlinear Systems, Marcel Dekker, New York, NY, USA, 1989.

[29] E. Massad and F. A. B. Coutinho, "Vectorial capacity, basic reproduction number, force of infection and all that: Formal notation to complete and adjust their classical concepts and equations," Memórias do Instituto Oswaldo Cruz, vol. 107, no. 4, pp. 564-567, 2012.

[30] O. Sharomi, C. N. Podder, A. B. Gumel, E. H. Elbasha, and J. Watmough, "Role of incidence function in vaccine-induced backward bifurcation in some HIV models," Mathematical Biosciences, vol. 210, no. 2, pp. 436-463, 2007.

[31] L. Fernandez Lopez, F. A. Bezerra Coutinho, M. Nascimento Burattini, and E. Massad, "Threshold conditions for infection persistence in complex host-vectors interactions," Comptes Rendus Biologies, vol. 325, no. 11, pp. 1073-1084, 2002.

[32] S. M. Raimundo, H. M. Yang, E. Venturino, and E. Massad, "Modeling the emergence of HIV-1 drug resistance resulting from antiretroviral therapy: Insights from theoretical and numerical studies," BioSystems, vol. 108, no. 1-3, pp. 1-13, 2012.
[33] A. Blumstein, J. Cohen, J. A. Roth, and C. A. Visher, Criminal Careers and Career Criminals, vol. I, The National Academies Press, Washington, DC, USA, 1986.

[34] J. F. MacLeod, P. Grove, and D. Farrington, Explaining Criminal Careers: Implications for Justice Policy, Oxford University Press, Oxford, UK, 2012. 


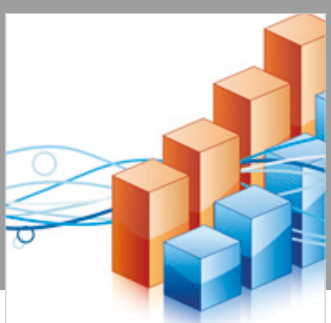

Advances in

Operations Research

\section{-n-m}
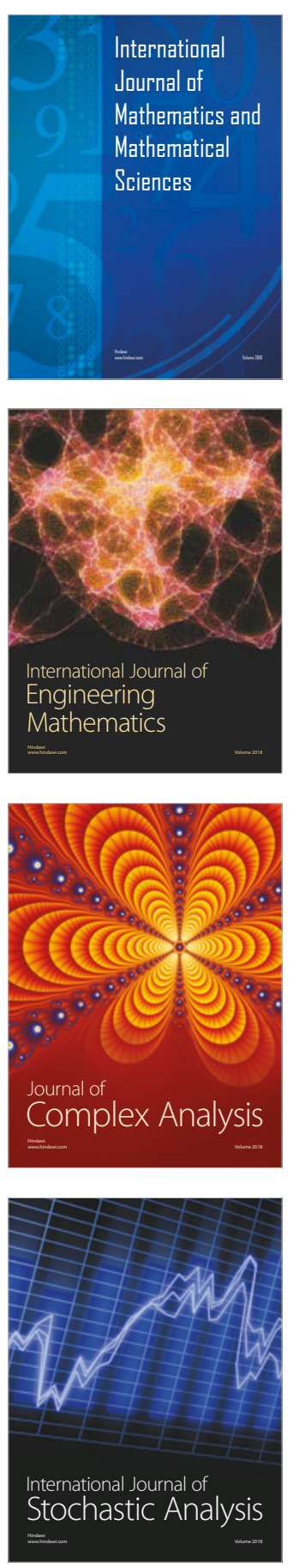
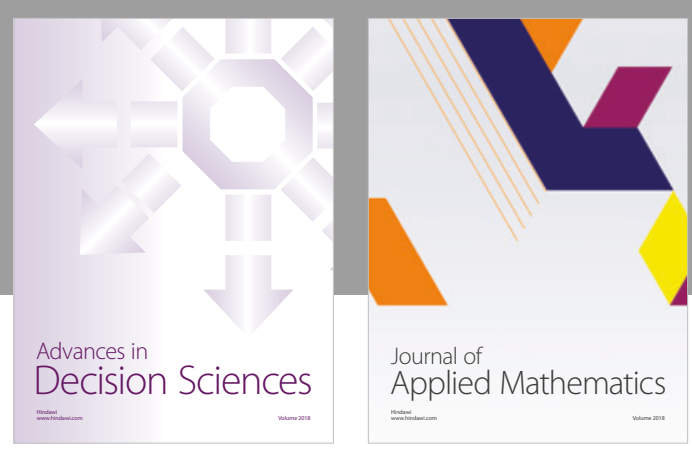

Journal of

Applied Mathematics
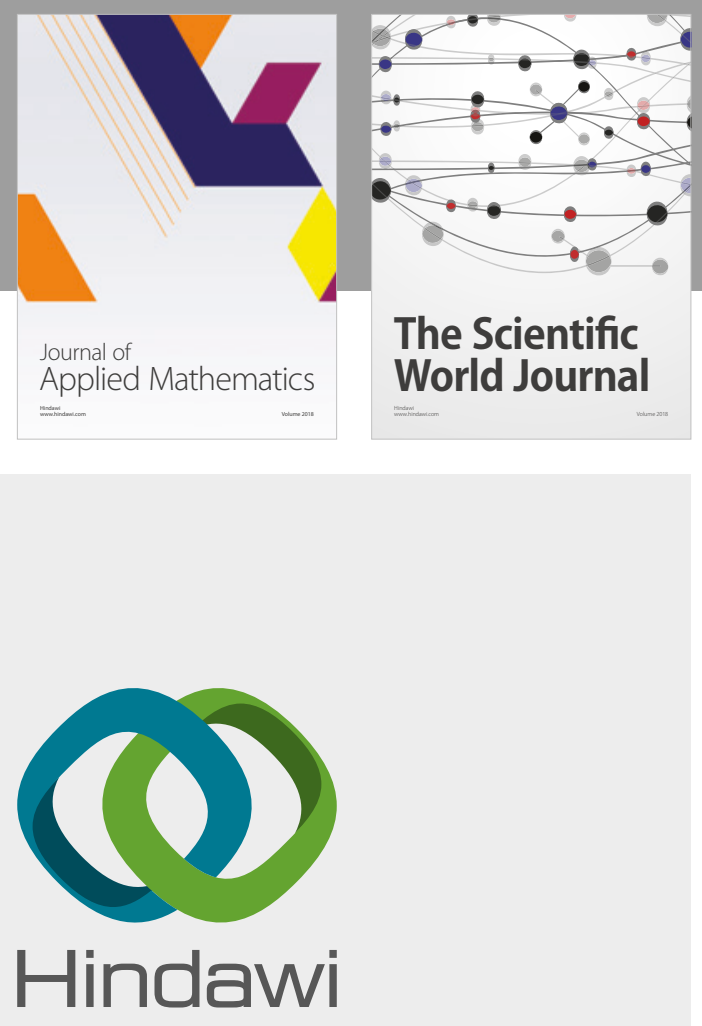

Submit your manuscripts at

www.hindawi.com

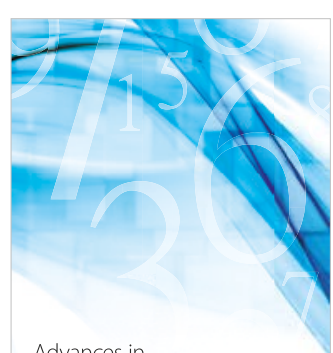

Advances in
Numerical Analysis
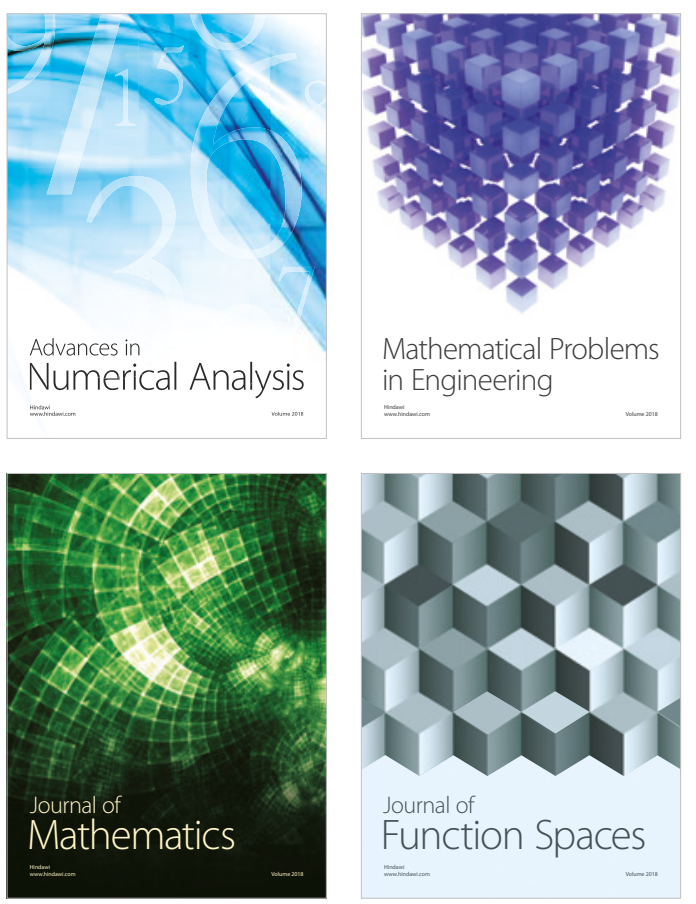

Mathematical Problems in Engineering

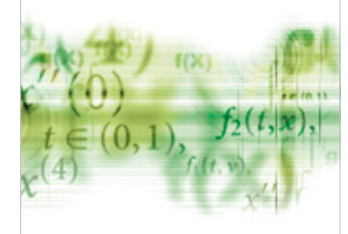

International Journal of

Differential Equations

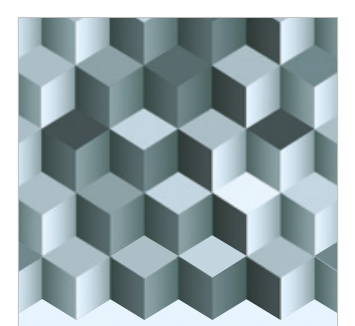

Journal of

Function Spaces

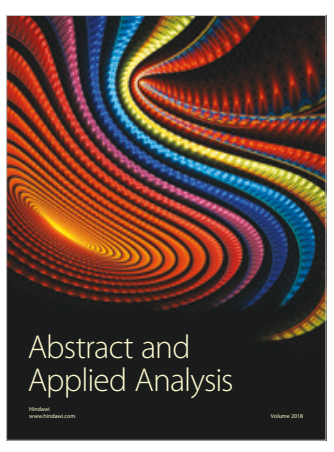

The Scientific

World Journal

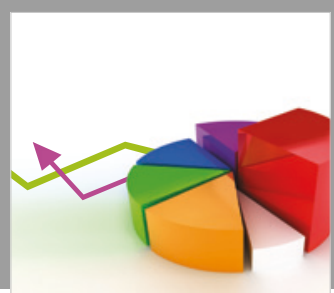

Journal of

Probability and Statistics
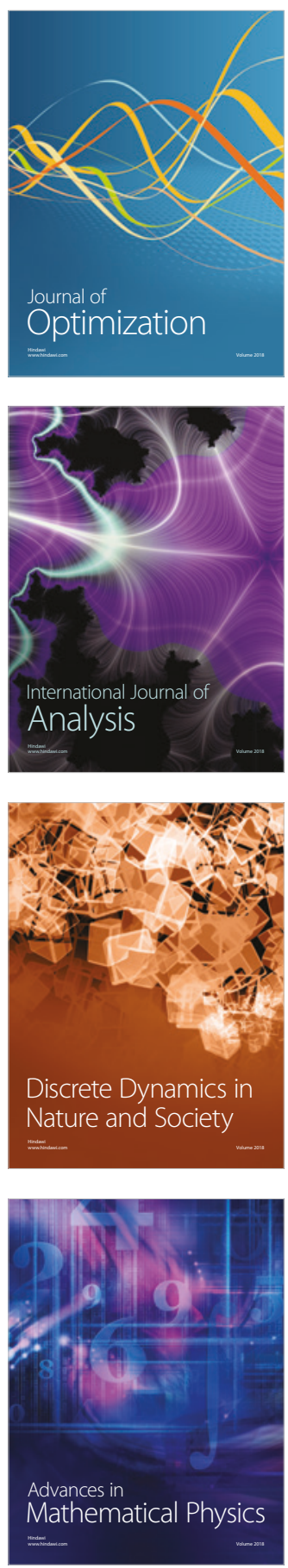